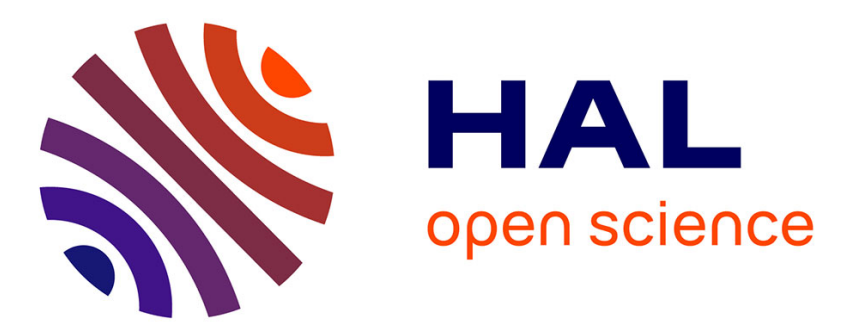

\title{
Reliability and survival analysis for drifting Markov models: modeling and estimation
}

Vlad Stefan Barbu, Nicolas Vergne

\section{To cite this version:}

Vlad Stefan Barbu, Nicolas Vergne. Reliability and survival analysis for drifting Markov models: modeling and estimation. Methodology and Computing in Applied Probability, 2018, 10.1007/s11009018-9682-8 . hal-02337216

HAL Id: hal-02337216

https://hal-normandie-univ.archives-ouvertes.fr/hal-02337216

Submitted on 29 Oct 2019

HAL is a multi-disciplinary open access archive for the deposit and dissemination of scientific research documents, whether they are published or not. The documents may come from teaching and research institutions in France or abroad, or from public or private research centers.
L'archive ouverte pluridisciplinaire HAL, est destinée au dépôt et à la diffusion de documents scientifiques de niveau recherche, publiés ou non, émanant des établissements d'enseignement et de recherche français ou étrangers, des laboratoires publics ou privés. 


\title{
Reliability and survival analysis for drifting Markov models: modeling and estimation
}

\author{
Vlad Stefan Barbu • Nicolas Vergne
}

Received: date / Accepted: date

\begin{abstract}
In this work we focus on multi-state systems modeled by means of a particular class of non-homogeneous Markov processes introduced in [37], called drifting Markov processes. The main idea behind this type of processes is to consider a non-homogeneity that is "smooth", of a known shape. More precisely, the Markov transition matrix is assumed to be a linear (polynomial) function of two (several) Markov transition matrices. For this class of systems, we first obtain explicit expressions for reliability/survival indicators of drifting Markov models, like reliability, availability, maintainability and failure rates. Then, under different statistical settings, we estimate the parameters of the model, obtain plug-in estimators of the associated reliability/survival indicators and investigate the consistency of the estimators. The quality of the proposed estimators and the model validation is illustrated through numerical experiments.
\end{abstract}

Keywords Drifting Markov chains $\cdot$ Multi-state systems $\cdot$ Reliability theory $\cdot$ Survival analysis $\cdot$ Estimation $\cdot$ Asymptotic properties

Mathematics Subject Classification (2000) 60J10 - 60K15 - 90B25 - 62N02 - 62F12

\section{Introduction}

In this work we focus on multi-state systems (MSS) modeled by means of particular class of non-homogeneous Markov processes introduced in [37], called drifting Markov processes. More specifically, we consider systems with a finite number of states, say $s$ states, $\{1, \ldots, s\}$. These different states of such a system usually represent different levels of performance. For instance, state "1" can be seen as being associated with the nominal performance of the system, state "s" can be seen as being associated with the total failure, while the other states have intermediate performance levels.

\section{V.S. Barbu}

Université de Rouen, Laboratoire de Mathématiques Raphaël Salem, UMR 6085, Avenue de l'Université, BP.12, F76801 Saint-Étienne-duRouvray, France

Tel.: +33232955231

Fax: +33232955286

E-mail: barbu@univ-rouen.fr

N. Vergne

Université de Rouen, Laboratoire de Mathématiques Raphaël Salem, UMR 6085, Avenue de l'Université, BP.12, F76801 Saint-Étienne-duRouvray, France

E-mail: nicolas.vergne@univ-rouen.fr 
Note that a hypothesis used in many mathematical models built as modeling tools for real applications is the homogeneity with respect to time.

On the one hand, it is clear that in many applications this homogeneity is inappropriate, at least for two reasons: first, there is often a non-homogeneity (or heterogeneity) intrinsic to the evolution of the systems; second, we can have interactions between the system and the environment, that can generate a non-homogeneity in the functioning of the system.

On the other hand, from a practical point of view, considering general non-homogeneous processes could be inappropriate, as the estimation procedures could be not tractable in practice. A possible solution is to consider a non-homogeneity that is "smooth", controlled, of a known shape. An example of this type in a Markov framework consists in the so called drifting Markov chains, introduced in [37]. For these processes, the Markov transition matrix is a linear (polynomial) function/mixture of two (several) Markov transition matrices. In other words, instead of fitting a transition matrix on a whole sequence of observations (homogeneous Markov model) or different transition matrices on different homogeneous parts of the observation (like we do in hidden Markov models), we allow the transition matrix to vary (to drift) from the beginning to the end of the observed phenomenon. Consequently, at each position, we obtain a different transition matrix, but with a controlled, known shape. These models are thus constrained non-homogeneous Markov models. Thus we obtain the desired "smooth" non-homogeneity. In this paper, we focus on polynomial drift ans associated reliability measures and estimation techniques. To the best of our knowledge, the use of such models in reliability/survival studies is a completely new approach.

Note that the type of models we propose is appropriate for modeling phenomena that evolve somehow smooth along time. On the one hand, it is worth to stress that this evolution is not only in one way, for instance aging evolution. The polynomial drift allows the models to take several possible shapes. On the other hand, if one needs piecewise homogeneity with abrupt transitions between the homogeneous zone, one possible setting is furnished by hidden Markov or hidden semi-Markov processes (see, e.g., [26], [9], [6] for general references on hidden Markov and semi-Markov models).

A remark needs to be done here. It is obvious that the family of stochastic models we propose in this article is a particular case of a non-homogeneous Markov chain. Consequently, using a general non-homogeneous Markov chain could be seen as a better modeling approach (and really could be in some situations). Nonetheless, note that the number of parameters of a (fully) non-homogeneous Markov chain could be huge as compared to the number of parameters of our models: two (for linear drift) or several (i.e., few) Markov transition matrices (for polynomial drift). Moreover, in order to be able to estimate them all, one needs an extremely huge number of copies of same type of trajectories (at every time $k$, we need an important or at least reasonable number of observations of transitions from any state to any another state).

The work developed along this paper belongs to MSS reliability methods, that have received a substantial amount of attention in the last decades. For theoretical advances and significant applications in MSS reliability theory in recent years, the reader is referred to [17], [23], [25] and [28].

Typical tools for investigating the evolution and performance of such systems are the Markov and semi-Markov processes. For general references on Markov processes and applications, as well as associated reliability topics, in continuous or discrete time, with corresponding estimation topics, one can see, e. g., [32], [33], [10], [35], [36], [12], [31]. Semi-Markov processes generalize renewal processes as well as Markov jump processes by allowing general distributions for sojourn times; this is the reason why the semi-Markov processes are more adapted for applications in general and for reliability studies in particular. One can see [18] for an introduction to continuous-time semi-Markov processes and [6] for a presentation of the corresponding discrete-time framework. 
For general references on continuous-time semi Markov systems, associated applications and reliability topics, one can see for example [19], [20], [29], [1], [27], [38], [2], [14], [16], [34]. Discrete-time semi-Markov modeling and estimation, and reliability computation and estimation can be found in [3], [4], [5], [11], [13]. A unified semi-Markov framework is described in [21].

This paper is structured as follows. We first introduce several types of drifting Markov models in the next section and present basic properties. We also obtain explicit expressions for reliability/survival indicators of drifting Markov models, like reliability, availability, maintainability and failure rates. Then, under different statistical settings, in Section 3 we estimate the parameters of the models, obtain plug-in estimators of the associated reliability/survival indicators and investigate the consistency of the estimators. The quality of the proposed estimators is also illustrated in Section 4, where numerical experiments are carried out.

\section{Drifting Markov models}

In this section we introduce several types of drifting Markov models, namely the linear drifting Markov chain of order 1 (Definition 1) and the polynomial drifting Markov chain of order $k$ (Definition 2), and present basic properties. Then we obtain explicit expressions for associated reliability/survival indicators, like reliability, availability, maintainability and failure rates; note that two distinct definitions of failure rates are considered.

\subsection{Definitions and notation}

Let us consider a random system with finite state space $E=\{1, \ldots, s\}, s<\infty$, and let $(\Omega, \mathcal{A}, \mathbb{P})$ be a probability space. All along this paper we assume that the time evolution of the system is governed by discrete-time stochastic processes defined on $(\Omega, \mathcal{A}, \mathbb{P})$ with values in $E$. Let $\Pi_{0}=\left(\Pi_{0}(u, v)\right)_{u, v \in E}$ and $\Pi_{1}=\left(\Pi_{1}(u, v)\right)_{u, v \in E}$ be two Markov transition matrices (of order 1) over a state space $E$.

Definition 1 (linear drifting Markov chain of order 1 and of length $n$ ) A sequence $X_{0}, X_{1}, \ldots, X_{n}$ with state space $E=\{1,2, \ldots, s\}$ is said to be a linear drifting Markov chain (of order 1) of length $n$ between the Markov transition matrices $\Pi_{0}$ and $\boldsymbol{\Pi}_{1}$ if the distribution of $X_{t}, t=1, \ldots, n$, is defined by

$$
\mathbb{P}\left(X_{t}=v \mid X_{t-1}=u, X_{t-2}, \ldots\right)=\Pi_{\frac{t}{n}}(u, v), u, v \in E
$$

where

$$
\Pi_{\frac{t}{n}}(u, v)=\left(1-\frac{t}{n}\right) \Pi_{0}(u, v)+\frac{t}{n} \Pi_{1}(u, v), u, v \in E
$$

Let us denote by $\boldsymbol{\alpha}=(\alpha(1), \ldots, \alpha(s))$ the initial distribution of the chain, that is the distribution of $X_{0}, \alpha(u)=\mathbb{P}\left(X_{0}=\right.$ $u$ ) for any state $u \in E$.

The following result, although straightforward, will be useful in the sequel.

Lemma 1 For a linear drifting Markov chain and $k_{1} \leq k_{2}, k_{1}, k_{2} \in \mathbb{N}$, we have

$$
\mathbb{P}\left(X_{k_{2}}=j \mid X_{k_{1}-1}=i\right)=\left(\prod_{l=k_{1}}^{k_{2}} \Pi_{\frac{l}{n}}\right)(i, j) .
$$




\section{Proof.}

The proof is straightforward and we omit it. In a most general case, it is given in Lemma 2

Note that one could consider a $k$ order linear drifting Markov chain, $k \in \mathbb{N}^{*}$, by allowing a dependence of order $k$ in Equation (1) and letting $\boldsymbol{\Pi}_{0}$ et $\boldsymbol{\Pi}_{1}$ be the transition probabilities of Markov chains of order $k$. Note also that the linear drifting can be generalized to polynomial drifting of a certain degree $d$. See [37] for more details on these points. Let us generalize linear drifting Markov model of order 1 to polynomial drifting Markov model of order $k$ and degree $d$. Let $\boldsymbol{\Pi}_{\frac{i}{d}}=\left(\boldsymbol{\Pi}_{\frac{i}{d}}\left(u_{1}, \ldots, u_{k}, v\right)\right)_{u_{1}, \ldots, u_{k}, v \in E}$ be $d$ Markov transition matrices (of order $k$ ) over a state space $E$.

Definition 2 (polynomial drifting Markov chain of order $k$ and of length $n$ ) A sequence $X_{0}, X_{1}, \ldots, X_{n}$ with state space $E=\{1,2, \ldots, s\}$ is said to be a polynomial drifting Markov chain of order $k$ and of length $n$ if the distribution of $X_{t}$, $t=1, \ldots, n$, is defined by

$$
\mathbb{P}\left(X_{t}=v \mid X_{t-1}=u_{k}, X_{t-2}=u_{k-1}, \ldots\right)=\Pi_{\frac{t}{n}}\left(u_{1}, \ldots, u_{k}, v\right), u_{1}, \ldots, u_{k}, v \in E
$$

where

$$
\boldsymbol{\Pi}_{\frac{t}{n}}\left(u_{1}, \ldots, u_{k}, v\right)=\sum_{i=0}^{d} A_{i}(t) \boldsymbol{\Pi}_{\frac{i}{d}}\left(u_{1}, \ldots, u_{k}, v\right), u_{1}, \ldots, u_{k}, v \in E,
$$

with $A_{i}$ polynomials of degree $d$ such as, for any $i, j \in\{0,1, \ldots, d\}, A_{i}\left(\frac{n j}{d}\right)=\mathbb{1}_{\{i=j\}}$.

Remark 1 Note that the coherence between notations implies the choice of the notation $\boldsymbol{\Pi}_{\frac{i}{d}}$. Indeed, for $t=n i / d$, we have $\Pi_{\frac{t}{n}}=\Pi_{\frac{i}{d}}$.

Remark 2 Polynomials $A_{i}$ are Lagrange polynomials : they are chosen to have $\boldsymbol{\Pi}_{\frac{t}{n}}$ a stochastic matrix for each $0 \leq t \leq n$. Indeed $\sum_{v \in E} \Pi_{\frac{t}{n}}\left(u_{1}, \ldots, u_{k}, v\right)=\sum_{i=0}^{d} A_{i}(t)=A(t)$ where $A$ is a polynomial of degree $d$ equal to one in $(d+1)$ points; then $A$ is a constant polynomial equal to one.

Example 1 (Polynomial drifting Markov model of degree 2) For a polynomial drifting Markov model of degree 2, we have

$$
\Pi_{\frac{t}{n}}(u, v)=A_{0}(t) \Pi_{0}(u, v)+A_{1}(t) \Pi_{\frac{1}{2}}(u, v)+A_{2}(t) \Pi_{1}(u, v)
$$

with $A_{0}, A_{1}, A_{2}$ polynomials of degree 2 and $\Pi_{0}, \Pi_{\frac{1}{2}}, \Pi_{1}$ parameter matrices.

We want to find $A_{0}, A_{1}, A_{2}$ such that $\Pi_{\frac{t}{n}}$ be a stochastic matrix and :

- for $t=0, \Pi_{\frac{t}{n}}=\Pi_{0}$, i.e. $A_{0}(0)=1, A_{1}(0)=0, A_{2}(0)=0$

- for $t=n / 2, \Pi_{\frac{t}{n}}=\Pi_{\frac{1}{2}}$, i.e. $A_{0}(n / 2)=0, A_{1}(n / 2)=1, A_{2}(n / 2)=0$

- for $t=n, \Pi_{\frac{t}{n}}=\Pi_{1}$, i.e. $A_{0}(n)=0, A_{1}(n)=0, A_{2}(n)=1$.

Solving these three systems we obtain

$$
\Pi_{\frac{t}{n}}(u, v)=\left(2 \frac{t^{2}}{n^{2}}-3 \frac{t}{n}+1\right) \Pi_{0}(u, v)+\left(-4 \frac{t^{2}}{n^{2}}+4 \frac{t}{n}\right) \Pi_{\frac{1}{2}}(u, v)+\left(2 \frac{t^{2}}{n^{2}}-\frac{t}{n}\right) \Pi_{1}(u, v) .
$$

Obviously, when dealing with a $k$ order Markov chain, we can always transform it into a Markov chain of order 1 , as it is stated in the following remark.

Remark 3 (From a Markov chain of order $k$ to a Markov chain of order 1 ) As it is well known, it is always possible to transform a Markov chain of order $k$ into a Markov chain of order 1 . We only have to change the state space: for an order $k$ Markov chain over a state space $E$ with transition $\pi$, we define an order 1 Markov chain over a state space $E^{\star}=E^{k}$ with transition $\pi^{\star}$ such as for $u \in E, v, v^{\prime} \in E^{k-1}$ and $w \in E, \pi^{\star}(u v ; v w)=\pi(u v ; w)$ and $\pi^{\star}\left(u v ; v^{\prime} w\right)=0$ if $v \neq v^{\prime}$. 
The following result is the analogous of Lemma 1 for a polynomial drifting Markov chain of order $k$ instead of a linear drifting Markov chain of order 1.

Lemma 2 For a polynomial drifting Markov chain of order $k$ and $k_{1}, k_{2} \in \mathbb{N}$, such that $k_{2}-k_{1} \geq k-1$, we have

$$
\mathbb{P}\left(X_{k_{2}}=j \mid X_{k_{1}-1}=i_{k}, \ldots, X_{k_{1}-k}=i_{1}\right)=\sum_{v_{1}, \ldots, v_{k-1} \in E}\left(\prod_{t=k_{1}}^{k_{2}} \Pi_{\frac{t}{n}}^{\star}\right)\left(\left(i_{1}, \ldots, i_{k}\right) ;\left(v_{1}, \ldots, v_{k-1}, j\right)\right)
$$

with $\boldsymbol{\Pi}_{\frac{t}{n}}^{\star}$ the corresponding transition matrix of the Markov chain of order 1 in the transformed alphabet $E^{\star}$ (see Remark 3).

Proof.

We immediately have

$$
\begin{aligned}
& \mathbb{P}\left(X_{k_{2}}=j \mid X_{k_{1}-1}=i_{k}, \ldots, X_{k_{1}-k}=i_{1}\right) \\
= & \sum_{\substack{i_{k_{1}}, i_{k_{1}+1}, \ldots, i_{k_{2}-1}\\
}} \mathbb{P}\left(X_{k_{2}}=j \mid X_{k_{2}-1}=i_{k_{2}-1}, \ldots, X_{k_{2}-k}=i_{k_{2}-k}\right) \\
= & \sum_{i_{k_{1}}, i_{k_{1}+1}, \ldots, i_{k_{2}-1}} \Pi_{\frac{k_{2}}{n}}\left(i_{k_{2}-k}, \ldots, i_{k_{2}-1}, j\right) \Pi_{\frac{k_{2}-1}{n}}\left(i_{k_{2}-k-1}, \ldots, i_{k_{2}-2}, i_{k_{2}-1}\right) \ldots \Pi_{\frac{k_{1}}{n}}\left(i_{1}, \ldots, i_{k}, i_{k_{1}}\right) \\
= & \sum_{v_{1}, \ldots, v_{k-1} \in E}\left(\prod_{t=k_{1}}^{k_{2}} \Pi_{\frac{t}{n}}^{\star}\right)\left(\left(i_{1}, \ldots, i_{k}\right) ;\left(v_{1}, \ldots, v_{k-1}, j\right)\right)
\end{aligned}
$$

where in the last line, we take into account all the possibilities for the $k$ states before state $j$ on the position $k_{2}$.

We would like to make some comments on the interest in practice and applicability of the drifting Markov models introduced in Definitions 1 and 2.

Remark 4 (practical interest of drifting Markov models) Note that the family of models introduced in this paper is fairly general, with a wide potential for real life applications. First, note that the classical Markov models of arbitrary order are particular cases of drifting Markov models. Second, in particular applications where the time evolution of the system is modelled by a Markov chain, it could make sense to assume that the evolution is different at the beginning of the lifetime, as compared to the end of the lifetime of the system. In such a situation, a linear drifting should be appropriate. Another typical situation is when the time evolution of a system could be split in three parts, corresponding to early failure/ stable failure/wear-out failure periods, respectively. In this case, a polynomial drifting Markov model of degree 2 could be appropriate. Surely, this decomposition in different epochs of the lifetime of a system can be considered not only for 2 or 3 epochs, but also for a different number, corresponding to different real situations depending on the application.

As mentioned in the Introduction, the drifting Markov models are particular non-homogeneous Markov models, with a controlled non-homogeneity. This is in fact one core point of the practical interest of drifting Markov models: it comes from the fact that we can adapt a parsimonious non-homogeneous model to a real application, with a reasonable number of parameters (as compared to a fully non-homogeneous model).

\subsection{Reliability and survival measures}

In order to undertake a reliability analysis of a repairable system modeled by a drifting Markov chain, let us assume that the state space of the system is partitioned into working and failure states, $E=U \cup D$, with $U \cap D=\emptyset$, where $U=\left\{1, \ldots, s_{1}\right\}$ are the working states and $D=\left\{s_{1}+1, \ldots, s\right\}$ the failure states of the system. Note that the states of 
$U$ can be seen as representing different operating modes or performance levels of the system (with state "1" associated with the nominal performance of the system), while the states of $D$ can represent different failure modes of the systems (with state "s" associated with the total failure).

According to this partition of the state space we partition any matrix of vector we are working with. For instance, we have

$$
\boldsymbol{\Pi}_{0}=\left(\begin{array}{cc}
U & D \\
\boldsymbol{\Pi}_{0}^{U U} & \boldsymbol{\Pi}_{\mathbf{0}}^{U D} \\
\boldsymbol{\Pi}_{0}^{D U} & \boldsymbol{\Pi}_{\mathbf{0}}^{D D}
\end{array}\right){ }_{D}^{U}, \quad \boldsymbol{\alpha}=\left(\begin{array}{cc}
\boldsymbol{\alpha}^{U} & \boldsymbol{\alpha}^{D}
\end{array}\right) .
$$

For a system starting to function at time $l=0$ (that is $\boldsymbol{\alpha}^{D}=\mathbf{0}$ ) the reliability at time $l \in \mathbb{N}$ is the probability that the system has functioned without failure in the period $[0, l]$.

Denoting by $T_{D}$ hitting time of subset $D$, called the lifetime of the system, i.e., $T_{D}:=\inf \left\{t \in \mathbb{N} ; \quad X_{t} \in D\right\}$, with inf $\emptyset:=$ $\infty$, the reliability at time $l \in \mathbb{N}$ is $R(l):=\mathbb{P}\left(T_{D}>l\right)=\mathbb{P}\left(X_{t} \in U, t=0, \ldots, l\right)$. The following results will present the expressions of reliability measures for the "simple" case of linear drifting Markov chains of order 1 (Propositions 1, 3, 5 and 7) and for the general case of polynomial drifting Markov chains of order $k$ (Propositions 2, 4, 6).

Proposition 1 For a linear drifting Markov chain of order $1\left(X_{t}\right)_{0 \leq t \leq n}$, the reliability at time $l, l \in \mathbb{N}$, is given by

$$
R(l)=\boldsymbol{\alpha}^{U} \prod_{t=1}^{l}\left(\left(1-\frac{t}{n}\right) \boldsymbol{\Pi}_{0}^{U U}+\frac{t}{n} \boldsymbol{\Pi}_{1}^{U U}\right) \mathbb{1}^{U}, \text { where } \mathbb{1}^{U}=(\underbrace{1, \cdots, 1}_{s_{1}})^{\top} .
$$

\section{Proof.}

We have

$$
R(l)=\mathbb{P}\left(X_{t} \in U, t=0, \ldots, l\right)=\sum_{i, j \in U} \mathbb{P}\left(X_{l}=j, X_{l-1} \in U, \ldots, X_{1} \in U \mid X_{0}=i\right) \mathbb{P}\left(X_{0}=i\right)=\sum_{i, j \in U} \alpha(i)\left(\prod_{t=1}^{l} \boldsymbol{\Pi}_{\frac{t}{n}}^{U U}\right)(i, j),
$$

where $\boldsymbol{\Pi}_{\frac{t}{n}}^{U U}:=\left(1-\frac{t}{n}\right) \boldsymbol{\Pi}_{0}^{U U}+\frac{t}{n} \boldsymbol{\Pi}_{1}^{U U}$ and we have used Lemma 1, with $\boldsymbol{\Pi}_{\frac{t}{n}}^{U U}, \boldsymbol{\Pi}_{1}^{U U}$ and $\boldsymbol{\Pi}_{0}^{U U}$ instead of $\boldsymbol{\Pi}_{\frac{t}{n}}, \boldsymbol{\Pi}_{1}$ and $\Pi_{0}$ respectively.

Proposition 2 For a polynomial drifting Markov chain of order $k\left(X_{t}\right)_{0 \leq t \leq n}$, the reliability at time $l, l \in \mathbb{N}$, is given by

$$
R(l)=\boldsymbol{\alpha}^{U} \sum_{j \in U}\left(\sum_{v_{1}, \ldots, v_{k-1} \in U} \prod_{t=k}^{l}\left(\boldsymbol{\Pi}_{\frac{t}{n}}^{U U}\right)^{\star}\right)\left(v_{1}, \ldots, v_{k-1}, j\right)
$$

\section{Proof.}

The proof is analogous to the proof of Proposition 1, using the passage from a Markov chain of order $k$ to a Markov chain of order 1 (cf. Remark 3). We have

$$
\begin{aligned}
R(l) & =\sum_{i_{0}, \ldots, i_{k-1}, j \in U} \mathbb{P}\left(X_{l}=j, X_{l-1} \in U, \ldots, X_{k} \in U \mid X_{k-1}=i_{k-1}, \ldots, X_{0}=i_{0}\right) \mathbb{P}\left(X_{k-1}=i_{k-1}, \ldots, X_{0}=i_{0}\right) \\
& =\sum_{i_{0}, \ldots, i_{k-1}, j \in U} \alpha\left(i_{0}, \ldots, i_{k-1}\right)\left(\sum_{v_{1}, \ldots, v_{k-1} \in U} \prod_{t=k}^{l}\left(\Pi_{\frac{t}{n}}^{U U}\right)^{\star}\left(\left(i_{0}, \ldots, i_{k-1}\right) ;\left(v_{1}, \ldots, v_{k-1}, j\right)\right)\right),
\end{aligned}
$$

where $\left(\boldsymbol{\Pi}_{\frac{t}{n}}^{U U}\right)^{\star}:=\left(1-\frac{t}{n}\right)\left(\boldsymbol{\Pi}_{0}^{U U}\right)^{\star}+\frac{t}{n}\left(\boldsymbol{\Pi}_{1}^{U U}\right)^{\star}$ and we have used Lemma 2 , with $\left(\boldsymbol{\Pi}_{\frac{t}{n}}^{U U}\right)^{\star},\left(\boldsymbol{\Pi}_{1}^{U U}\right)^{\star}$ and $\left(\boldsymbol{\Pi}_{0}^{U U}\right)^{\star}$ instead of $\boldsymbol{\Pi}_{\frac{t}{n}}^{\star}, \boldsymbol{\Pi}_{1}^{\star}$ and $\boldsymbol{\Pi}_{0}^{\star}$ respectively (that is to say that we considered the restriction of the initial $k$ order Markov chain to the subspace $U$, and, consequently, the associate restriction of the extended Markov chain of order 1). 
Let us now consider the pointwise (or instantaneous) availability; for any $l \in \mathbb{N}$, the availability at time $l$ it is defined as the probability that the system is in a working state at time $l$, independently of the fact that the system worked or not during the time interval $[0 ; l), A(l):=\mathbb{P}\left(X_{l} \in U\right)$.

Proposition 3 For a linear drifting Markov chain of order $1\left(X_{t}\right)_{0 \leq t \leq n}$, the pointwise (or instantaneous) availability at time $l, l \in \mathbb{N}$, is given by

$$
A(l)=\boldsymbol{\alpha} \prod_{t=1}^{l}\left(\left(1-\frac{t}{n}\right) \boldsymbol{\Pi}_{0}+\frac{t}{n} \boldsymbol{\Pi}_{1}\right) \mathbb{1}^{E, U}, \text { where } \mathbb{1}^{E, U}=(\underbrace{1, \cdots, 1}_{s_{1}}, \underbrace{0, \cdots, 0}_{s-s_{1}})^{\top} .
$$

\section{Proof.}

As in the proof of the expression of the reliability, we immediately have

$$
A(l)=\sum_{i \in E, j \in U} \mathbb{P}\left(X_{l}=j \mid X_{0}=i\right) \mathbb{P}\left(X_{0}=i\right)=\sum_{i \in E, j \in U} \alpha(i)\left(\prod_{t=1}^{l} \boldsymbol{\Pi}_{\frac{t}{n}}\right)(i, j) .
$$

Proposition 4 For a polynomial drifting Markov chain of order $k\left(X_{t}\right)_{0 \leq t \leq n}$, the pointwise (or instantaneous) availability at time $l, l \in \mathbb{N}$, is given by

$$
A(l)=\boldsymbol{\alpha} \sum_{j \in U}\left(\sum_{v_{1}, \ldots, v_{k-1} \in E} \prod_{t=k}^{l} \boldsymbol{\Pi}_{\frac{t}{n}}^{\star}\right)\left(v_{1}, \ldots, v_{k-1}, j\right) .
$$

\section{Proof.}

The proof is similar to the proof of Proposition 2.

A remark needs to be done here; for a similar remark in a discrete-time semi-Markov framework, see [6].

Remark 5 When computing reliability measures, one can consider either the case of ergodic (i.e., repairable) systems, or the case of non-ergodic (i.e., non-repairable) systems. For non-repairable systems, the set $D$ is absorbing; consequently, in this case the availability and reliability coincide and are given by Formula (6). Obviously, for repairable systems availability and reliability do not coincide, and one has to consider the different formulas presented above.

To conclude, although in this paper we consider ergodic (repairable) systems, explicit forms of the reliability indicators can be immediately obtained even in the non-ergodic (non-repairable) case.

Let us assume that we deal with a repairable system for which the failure occurs at time $l=0$ (in other words, we take $\boldsymbol{\alpha}^{U}=\mathbf{0}$ ). Its maintainability at time $k \in \mathbb{N}$ is the probability that the system is repaired up to time $l$, given that it has failed at time $l=0$. Let us denote by $T_{U}$ the first hitting time of subset $U$, called the duration of repair or repair time, that is, $T_{U}=\inf \left\{t \in \mathbb{N} ; X_{t} \in U\right\}$. Thus, the maintainability at time $l \in \mathbb{N}$ is defined by $M(l):=\mathbb{P}\left(T_{U} \leq l\right)=1-\mathbb{P}\left(T_{U}>\right.$ $l)=1-\mathbb{P}\left(X_{t} \in D, t=0, \ldots, l\right)$.

Proposition 5 For a linear drifting Markov chain of order $1\left(X_{t}\right)_{0 \leq t \leq n}$, the maintainability at time $l, l \in \mathbb{N}$, is given by

$$
M(l)=1-\boldsymbol{\alpha}^{D} \prod_{t=1}^{l}\left(\left(1-\frac{t}{n}\right) \boldsymbol{\Pi}_{0}^{D D}+\frac{t}{n} \boldsymbol{\Pi}_{1}^{D D}\right) \mathbb{1}^{D}, \text { where } \mathbb{1}^{D}=(\underbrace{1, \cdots, 1}_{s-s_{1}})^{\top} .
$$

\section{Proof.}

The proof is similar to the one of Proposition 1, exchanging the places of $U$ and $D$. 
Proposition 6 For a polynomial drifting Markov chain of order $k\left(X_{t}\right)_{0 \leq t \leq n}$, the maintainability at time $l, l \in \mathbb{N}$, is given by

$$
M(l)=1-\boldsymbol{\alpha}^{D} \sum_{j \in D}\left(\sum_{v_{1}, \ldots, v_{k-1} \in D} \prod_{t=k}^{l}\left(\boldsymbol{\Pi}_{\frac{t}{n}}^{D D}\right)^{\star}\right)\left(v_{1}, \ldots, v_{k-1}, j\right) .
$$

\section{Proof.}

The proof is similar to the proof of Proposition 2, exchanging the places of $U$ and $D$.

In the sequel we will be interested in the failure rate of the system. To this purpose, we will consider two different definitions of the failure rate: the first one is the classical failure rate introduced in [8] and usually considered for continuous time systems; this one will be called the BMP-failure rate and will be denoted by $\lambda(l), l \in \mathbb{N}$. The second one, proposed in [30], is a failure rate adapted to the work in discrete time; we will call it the $R G$-failure rate and denote it by $r(l), l \in \mathbb{N}$. The interested reader could see also [39] for detailed justification of the use of the RG-failure rate in discrete time, instead of the classical one. The use of this failure rate for discrete time Markov chains can be found in [32], while for discrete time semi-Markov chains can be found in [4] and [6].

Definition 3 (failure rates; cf. [8], [30], [39]) Let us consider a discrete time system starting to work at time $l=0$ (that is $\left.\boldsymbol{\alpha}^{D}=\mathbf{0}\right)$.

1. The BMP-failure rate at time $l \in \mathbb{N}$ is the conditional probability that the failure of the system occurs at time $l$, given that the system has worked until time $l-1, i . e$. ,

$$
\lambda(l):=\mathbb{P}\left(T_{D}=l \mid T_{D} \geq l\right)= \begin{cases}1-\frac{R(l)}{R(l-1)}, & \text { if } R(l-1) \neq 0 \\ 0, & \text { otherwise }\end{cases}
$$

2. The RG-failure rate at time $l \in \mathbb{N}$ is defined by

$$
r(l):=\left\{\begin{array}{l}
-\ln \frac{R(l)}{R(l-1)}, \text { if } l \geq 1, \\
-\ln R(0), \quad \text { if } l=0,
\end{array}\right.
$$

for $R(l) \neq 0$. If $R(l)=0$, we set $r(l):=0$.

Note that the two failure rates are related by $r(l)=-\ln (1-\lambda(l)), l \in \mathbb{N}$.

Taking into account the expression of the reliability of a drifting Markov chain given in Proposition 1, we immediately obtain the expression of the failure rates for a linear drifting Markov chain of order 1 . The general case can be immediately written using Proposition 2.

Proposition 7 For a linear drifting Markov chain of order $1\left(X_{t}\right)_{0 \leq t \leq n}$, the BMP-failure rate $\lambda(l)$ and the RG-failure rate $r(l)$ are given by:

$$
\begin{gathered}
\lambda(l)=\left\{\begin{array}{cc}
1-\frac{\boldsymbol{\alpha}^{U} \prod_{t=1}^{l}\left(\left(1-\frac{t}{n}\right) \boldsymbol{\Pi}_{0}^{U U}+\frac{t}{n} \boldsymbol{\Pi}_{1}^{U U}\right) \mathbb{1}^{U}}{\boldsymbol{\alpha}^{U} \prod_{t=1}^{l-1}\left(\left(1-\frac{t}{n}\right) \boldsymbol{\Pi}_{0}^{U U}+\frac{t}{n} \boldsymbol{\Pi}_{1}^{U U}\right) \mathbb{1}^{U}}, & \text { if } R(l-1) \neq 0, \\
0, & \text { otherwise, }
\end{array}\right. \\
r(l)=\left\{\begin{array}{ll}
-\ln \frac{\boldsymbol{\alpha}^{U} \prod_{t=1}^{l}\left(\left(1-\frac{t}{n}\right) \boldsymbol{\Pi}_{0}^{U U}+\frac{t}{n} \boldsymbol{\Pi}_{1}^{U U}\right) \mathbb{1}^{U}}{\boldsymbol{\alpha}^{U} \prod_{t=1}^{l-1}\left(\left(1-\frac{t}{n}\right) \boldsymbol{\Pi}_{0}^{U U}+\frac{t}{n} \boldsymbol{\Pi}_{1}^{U U}\right) \mathbb{1}^{U}}, & \text { if } l \geq 1, \\
-\ln R(0), & \text { if } l=0,
\end{array}\right)^{\top} .
\end{gathered}
$$

for $R(l) \neq 0$. If $R(l)=0$, we set $r(l):=0$. 


\section{Estimation of drifting Markov models}

The purpose of this section is twofold: first, continuing the direction developed in [37], we will consider different types of data for which the estimators of the characteristics of a drifting Markov chain will be derived. Second, we will estimate the associated reliability indicators.

For estimation purposes we first consider two types of data: the first one consists in one sample path of a linear drifting Markov chain; the second one consists in several sample paths of the same fixed length. Then we consider various types of data that can occur in practice and we adapt the estimation procedures that we have previously obtained in order to get estimators of the drifting Markov models in these cases. These different types of data arise from three ingredients: an observed sample path varying between two Markov transition matrices could be complete or incomplete; we can have at our disposal either one or several sample paths; several observed sample paths could be of the same length or of different length. The possible types of data that we take into account are the following:

A. One can observe a sample path, that will be denoted by $\mathcal{H}(m, n):=\left(X_{0}, X_{1}, \ldots, X_{m}\right)$, where $m$ denotes the length of the sample path and $n$ the length of the drifting Markov chain. Two cases can be considered:

A1 : $m=n$ (a complete sample path);

A2 : $m \leq n$ (an incomplete sample path).

B. One can also observe $H$ i.i.d. sample paths, $\mathcal{H}_{i}\left(m_{i}, n_{i}\right), i=1, \ldots, H$. Four cases can be considered here:

B1 : $m_{i}=n_{i}=n$ for all $i=1, \ldots, H$ (complete sample paths of drifting Markov chains of the same length) ;

B2 $: n_{i}=n$ for all $i=1, \ldots, H$ (incomplete sample paths of drifting Markov chains of the same length);

B3 : $m_{i}=n_{i}$ for all $i=1, \ldots, H$ (complete sample paths of drifting Markov chains of different lengths) ;

B4 : $m_{i} \leq n_{i}$ for all $i=1, \ldots, H$ (incomplete sample paths of drifting Markov chains of different lengths).

Notation 1 We introduce the following notations:

$P_{1}(H, \boldsymbol{m}, \boldsymbol{n})=\sum_{t=1}^{m} \sum_{h=1}^{H} \mathbb{1}_{\left\{X_{t-1}^{h}=u\right\}}\left(\frac{t}{n}\right)^{2}, P_{2}(H, \boldsymbol{m}, \boldsymbol{n})=\sum_{t=1}^{m} \sum_{h=1}^{H} \mathbb{1}_{\left\{X_{t-1}^{h}=u, X_{t}^{h}=v\right\}}\left(1-\frac{t}{n}\right)$,

$P_{3}(H, \boldsymbol{m}, \boldsymbol{n})=\sum_{t=1}^{m} \sum_{h=1}^{H} \mathbb{1}_{\left\{X_{t-1}^{h}=u\right\}}\left(1-\frac{t}{n}\right)\left(\frac{t}{n}\right), P_{4}(H, \boldsymbol{m}, \boldsymbol{n})=\sum_{t=1}^{m} \sum_{h=1}^{H} \mathbb{1}_{\left\{X_{t-1}^{h}=u, X_{t}^{h}=v\right\}}\left(\frac{t}{n}\right)$,

$P_{5}(H, \boldsymbol{m}, \boldsymbol{n})=\sum_{t=1}^{m} \sum_{h=1}^{H} \mathbb{1}_{\left\{X_{t-1}^{h}=u\right\}}\left(1-\frac{t}{n}\right)^{2}$, with $\boldsymbol{m}=\left(m_{1}, \ldots, m_{H}\right)$ and $\boldsymbol{n}=\left(n_{1}, \ldots, n_{H}\right)$.

3.1 Estimation of drifting Markov models: the usual types of data

From [37], we have the following result.

Proposition 8 For $\left(X_{0}, X_{1}, \ldots, X_{n}\right)$ a sample path of a linear drifting Markov chain between two Markov transition matrices (of order 1) $\Pi_{0}$ and $\Pi_{1}$, for any states $u, v \in E$, the least square estimators of $\Pi_{0}(u, v)$ and $\Pi_{1}(u, v)$ are given by:

$$
\begin{aligned}
\widehat{\Pi}_{0 ; n}(u, v) & =\frac{P_{1}(1, n, n) P_{2}(1, n, n)-P_{3}(1, n, n) P_{4}(1, n, n)}{P_{5}(1, n, n) P_{1}(1, n, n)-P_{3}(1, n, n)^{2}} \\
\widehat{\Pi}_{1 ; n}(u, v) & =\frac{P_{5}(1, n, n) P_{4}(1, n, n)-P_{3}(1, n, n) P_{2}(1, n, n)}{P_{5}(1, n, n) P_{1}(1, n, n)-P_{3}(1, n, n)^{2}} .
\end{aligned}
$$

Consequently, from Equation (2), for any $u, v \in E$ and $t=1, \ldots, n$, the estimator of $\Pi_{\frac{t}{n}}(u, v)$ is given by

$$
\widehat{\Pi}_{\frac{t}{n}}(u, v)=\left(1-\frac{t}{n}\right) \widehat{\Pi}_{0 ; n}(u, v)+\frac{t}{n} \widehat{\Pi}_{1 ; n}(u, v),
$$


with $\widehat{\Pi}_{0 ; n}(u, v)$ and $\widehat{\Pi}_{1 ; n}(u, v)$ given in (15) and (16), respectively.

Second, let us now consider $H$ i.i.d. sample paths of a linear drifting Markov chain $\left(X_{k}\right)_{0 \leq k \leq n}, \mathcal{H}_{i}(m, n), i=1, \ldots, H$. That is, we assume that the sample paths have the same length $n$ and that the linear drift within the $H$ sample paths takes place between the same two Markov transition matrices (of order 1) $\Pi_{0}$ and $\Pi_{1}$ (framework B1).

Proposition 9 Under this setting, starting with $H$ sample paths of a linear drifting Markov chain between two Markov transition matrices (of order 1) $\Pi_{0}$ and $\Pi_{1}$, for any states $u, v \in E$, the estimators of $\Pi_{0}(u, v)$ and $\Pi_{1}(u, v)$ are given by:

$$
\begin{aligned}
\widehat{\Pi}_{0 ;(n, H)}(u, v) & =\frac{P_{1}(H, \boldsymbol{n}, \boldsymbol{n}) P_{2}(H, \boldsymbol{n}, \boldsymbol{n})-P_{3}(H, \boldsymbol{n}, \boldsymbol{n}) P_{4}(H, \boldsymbol{n}, \boldsymbol{n})}{P_{5}(H, \boldsymbol{n}, \boldsymbol{n}) P_{1}(H, \boldsymbol{n}, \boldsymbol{n})-P_{3}(H, \boldsymbol{n}, \boldsymbol{n})^{2}}, \\
\widehat{\Pi}_{1 ;(n, H)}(u, v) & =\frac{P_{5}(H, \boldsymbol{n}, \boldsymbol{n}) P_{4}(H, \boldsymbol{n}, \boldsymbol{n})-P_{3}(H, \boldsymbol{n}, \boldsymbol{n}) P_{2}(H, \boldsymbol{n}, \boldsymbol{n})}{P_{5}(H, \boldsymbol{n}, \boldsymbol{n}) P_{1}(H, \boldsymbol{n}, \boldsymbol{n})-P_{3}(H, \boldsymbol{n}, \boldsymbol{n})^{2}},
\end{aligned}
$$

with $\boldsymbol{n}=\left(n_{1}, \ldots, n_{H}\right)=(n, \ldots, n)$.

\section{Proof.}

As in [37], we minimize a quadratic form of the different parameters which is the sum of "prediction errors". For each sequence $h$, at each position $t$ in the sequence, knowing $X_{t-1}=u$, we want $\Pi_{\frac{t}{n}}(u, v)$ to be the nearest possible to 1 if $X_{t}=v$ or the nearest possible to 0 if $X_{t} \neq v$. We minimize the sum of squares of the errors

$$
\sum_{h=1}^{H} \mathbb{1}_{\left\{X_{t-1}^{h}=u\right\}} \Pi_{\frac{t}{n}}(u, v)-\sum_{h=1}^{H} \mathbb{1}_{\left\{X_{t-1}^{h}=u ; X_{t}^{h}=v\right\}} .
$$

We choose a quadratic distance and then we minimize the following function:

$$
\sum_{t=1}^{n} \sum_{u \in \mathcal{A}^{k}} \sum_{v \in \mathcal{A}} \sum_{h=1}^{H} \mathbb{1}_{\left\{X_{t-1}^{h}=u\right\}}\left(\Pi_{\frac{t}{n}}(u, v)-\mathbb{1}_{\left\{X_{t}^{h}=v\right\}}\right)^{2} .
$$

Then, we obtain (18) and (19) (similarly as (15) and (16) in Proposition 8).

Consequently, from Equation (2), for any $u, v \in E$ and $t=1, \ldots, n$, the estimator of $\Pi_{\frac{t}{n}}(u, v)$ is given by

$$
\widehat{\Pi}_{\frac{t}{n} ; H}(u, v)=\left(1-\frac{t}{n}\right) \widehat{\Pi}_{0 ;(n, H)}(u, v)+\frac{t}{n} \widehat{\Pi}_{1 ;(n, H)}(u, v),
$$

with $\widehat{\Pi}_{0 ;(n, H)}(u, v)$ and $\widehat{\Pi}_{1 ;(n, H)}(u, v)$ given in (18) and (19), respectively.

Theorem 1 For a linear drifting Markov chain $\left(X_{k}\right)_{0 \leq k \leq n}$ between two Markov transition matrices (of order 1) $\boldsymbol{\Pi}_{0}$ and $\boldsymbol{\Pi}_{1}$, for any states $u, v \in E$ and time $t, t=1, \ldots, n$, the estimators of $\Pi_{0}(u, v), \Pi_{1}(u, v)$ and $\Pi_{\frac{t}{n}}(u, v)$ given in Equations (18), (19) and (20), respectively, are strongly consistent, i.e.,

$$
\widehat{\Pi}_{0 ;(n, H)}(u, v) \underset{H \rightarrow \infty}{\stackrel{a . s .}{\longrightarrow}} \Pi_{0}(u, v), \widehat{\Pi}_{1 ;(n, H)}(u, v) \underset{H \rightarrow \infty}{\stackrel{a . s .}{\longrightarrow}} \Pi_{1}(u, v), \widehat{\Pi}_{\frac{t}{n} ; H}(u, v) \underset{H \rightarrow \infty}{\stackrel{a . s .}{\longrightarrow}} \Pi_{\frac{t}{n}}(u, v) .
$$

\section{Proof.}

First, for any states $u, v \in E$ and time $t=1, \ldots, n$, note that from the Strong law of large numbers and from the definition of a drifting Markov chain, we immediately have

$$
\frac{1}{H} \sum_{h=1}^{H} \mathbb{1}_{\left\{X_{t-1}^{h}=u\right\}} \underset{H \rightarrow \infty}{\stackrel{a . s .}{\longrightarrow}} \mathbb{P}\left(X_{t-1}=u\right), \frac{1}{H} \sum_{h=1}^{H} \mathbb{1}_{\left\{X_{t-1}^{h}=u, X_{t}^{h}=v\right\}} \underset{H \rightarrow \infty}{\stackrel{a . s .}{\longrightarrow}} \mathbb{P}\left(X_{t-1}=u\right) \Pi_{\frac{t}{n}}(u, v) .
$$


Second, we obtain the following limits

$$
\begin{gathered}
\widehat{\Pi}_{0 ;(n, H)}(u, v) \underset{H \rightarrow \infty}{\stackrel{a . s .}{\longrightarrow}} \frac{\sum_{\left(t_{1}, t_{2}\right) \in \llbracket 1, n \rrbracket^{2}, t_{1} \neq t_{2}} \mathbb{P}\left(X_{t_{1}-1}=u\right) \mathbb{P}\left(X_{t_{2}-1}=u\right) \Pi_{\frac{t_{2}}{n}}(u, v)\left(\frac{t_{1}}{n}\right)\left(\frac{t_{1}}{n}\left(1-\frac{t_{2}}{n}\right)-\frac{t_{2}}{n}\left(1-\frac{t_{1}}{n}\right)\right)}{\sum_{\left(t_{1}, t_{2}\right) \in \llbracket 1, n \rrbracket^{2}, t_{1} \neq t_{2}} \mathbb{P}\left(X_{t_{1}-1}=u\right) \mathbb{P}\left(X_{t_{2}-1}=u\right)\left(1-\frac{t_{1}}{n}\right)\left(\frac{t_{2}}{n}\right)\left(\frac{t_{2}}{n}-\frac{t_{1}}{n}\right)} \\
=\frac{\sum_{\left(t_{1}, t_{2}\right) \in \llbracket 1, n \rrbracket^{2}, t_{1} \neq t_{2}} \mathbb{P}\left(X_{t_{1}-1}=u\right) \mathbb{P}\left(X_{t_{2}-1}=u\right) \Pi_{0}(u, v)\left(1-\frac{t_{2}}{n}\right)\left(\frac{t_{1}}{n}\right)\left(\frac{t_{1}}{n}-\frac{t_{2}}{n}\right)}{\sum_{\left(t_{1}, t_{2}\right) \in \llbracket 1, n \rrbracket^{2}, t_{1} \neq t_{2}} \mathbb{P}\left(X_{t_{1}-1}=u\right) \mathbb{P}\left(X_{t_{2}-1}=u\right)\left(1-\frac{t_{1}}{n}\right)\left(\frac{t_{2}}{n}\right)\left(\frac{t_{2}}{n}-\frac{t_{1}}{n}\right)} \\
\sum_{\left(t_{1}, t_{2}\right) \in \llbracket 1, n \rrbracket^{2}, t_{1} \neq t_{2}} \mathbb{P}\left(X_{t_{1}-1}=u\right) \mathbb{P}\left(X_{t_{2}-1}=u\right) \Pi_{1}(u, v)\left(\frac{t_{2}}{n}\right)\left(\frac{t_{1}}{n}\right)\left(\frac{t_{1}}{n}-\frac{t_{2}}{n}\right) \\
\mathbb{P}\left(X_{t_{1}-1}=u\right) \mathbb{P}\left(X_{t_{2}-1}=u\right)\left(1-\frac{t_{1}}{n}\right)\left(\frac{t_{2}}{n}\right)\left(\frac{t_{2}}{n}-\frac{t_{1}}{n}\right)
\end{gathered}
$$

Thus $\widehat{\Pi}_{0 ;(n, H)}(u, v) \underset{H \rightarrow \infty}{\stackrel{a . s .}{\longrightarrow}} \Pi_{0}$.

$$
\begin{gathered}
\widehat{\Pi}_{1 ;(n, H)}(u, v) \underset{H \rightarrow \infty}{\text { a.s. }} \rightarrow \frac{\sum_{\left(t_{1}, t_{2}\right) \in \llbracket 1, n \rrbracket^{2}, t_{1} \neq t_{2}} \mathbb{P}\left(X_{t_{1}-1}=u\right) \mathbb{P}\left(X_{t_{2}-1}=u\right) \Pi_{\frac{t_{2}}{n}}(u, v)\left(1-\frac{t_{1}}{n}\right)\left(\frac{t_{2}}{n}-\frac{t_{1}}{n}\right)}{\sum_{\left(t_{1}, t_{2}\right) \in \llbracket 1, n \rrbracket^{2}, t_{1} \neq t_{2}} \mathbb{P}\left(X_{t_{1}-1}=u\right) \mathbb{P}\left(X_{t_{2}-1}=u\right)\left(1-\frac{t_{1}}{n}\right)\left(\frac{t_{2}}{n}\right)\left(\frac{t_{2}}{n}-\frac{t_{1}}{n}\right)} \\
=\frac{\sum_{\left(t_{1}, t_{2}\right) \in \llbracket 1, n \rrbracket^{2}, t_{1} \neq t_{2}} \mathbb{P}\left(X_{t_{1}-1}=u\right) \mathbb{P}\left(X_{t_{2}-1}=u\right) \Pi_{0}(u, v)\left(1-\frac{t_{2}}{n}\right)\left(1-\frac{t_{1}}{n}\right)\left(\frac{t_{2}}{n}-\frac{t_{1}}{n}\right)}{\sum_{\left(t_{1}, t_{2}\right) \in \llbracket 1, n \rrbracket^{2}, t_{1} \neq t_{2}} \mathbb{P}\left(X_{t_{1}-1}=u\right) \mathbb{P}\left(X_{t_{2}-1}=u\right)\left(1-\frac{t_{1}}{n}\right)\left(\frac{t_{2}}{n}\right)\left(\frac{t_{2}}{n}-\frac{t_{1}}{n}\right)} \\
=\frac{\left(t_{1}, t_{2}\right) \in \llbracket 1, n \rrbracket^{2}, t_{1} \neq t_{2}}{\sum_{\left(t_{1}, t_{2}\right) \in \llbracket 1, n \rrbracket^{2}, t_{1} \neq t_{2}} \mathbb{P}\left(X_{t_{1}-1}=u\right) \mathbb{P}\left(X_{t_{2}-1}=u\right) \Pi_{1}(u, v)\left(\frac{t_{2}}{n}\right)\left(1-\frac{t_{1}}{n}\right)\left(\frac{t_{2}}{n}-\frac{t_{1}}{n}\right)}
\end{gathered}
$$

Thus $\widehat{\Pi}_{1 ;(n, H)}(u, v) \underset{H \rightarrow \infty}{\stackrel{a . s .}{\longrightarrow}} \Pi_{1}$.

In the case of $H$ i.i.d. sample paths of a linear drifting Markov chain $\left(X_{k}\right)_{0 \leq k \leq n}$, it is immediate to show that the MLE of the initial distribution of the chain is given by

$$
\widehat{\alpha}_{H}(i)=\frac{N_{i, 0}^{(H)}}{H},
$$

where $N_{i, 0}^{(H)}:=\sum_{l=1}^{H} \mathbb{1}_{\left\{X_{0}^{l}=i\right\}}$ represents the number of sample paths starting in state $i$ among the $H$ sample paths.

Proposition 10 Let us note $\mathbb{1}_{u}=\mathbb{1}_{\left\{X_{t-k} \ldots X_{t-1}=u_{1} \ldots u_{k}\right\}}$ and $\mathbb{1}_{u v}=\mathbb{1}_{\left\{X_{t-k} \ldots X_{t-1}=u_{1} \ldots u_{k}, X_{t}=v\right\}}$. For $\left(X_{0}, X_{1}, \ldots, X_{n}\right)$ a sample path of a polynomial drifting Markov chain of order $k$ and degree $d$, for any states $u_{1}, \ldots, u_{k}, v \in E$, the estimators of $\Pi_{\frac{i}{d}}\left(u 1, \ldots u_{k}, v\right)$ are given by solving the following linear system

$$
\left(\begin{array}{ccc}
\sum_{t=k}^{n} \mathbb{1}_{u} A_{0}(t) A_{0}(t) & \cdots & \sum_{t=k}^{n} \mathbb{1}_{u} A_{0}(t) A_{d}(t) \\
\vdots & \vdots \\
\sum_{t=k}^{n} \mathbb{1}_{u} A_{d}(t) A_{0}(t) & \cdots & \sum_{t=k}^{n} \mathbb{1}_{u} A_{d}(t) A_{d}(t)
\end{array}\right)\left(\begin{array}{c}
\widehat{\Pi_{0 ; n}}\left(u_{1}, \ldots, u_{k}, v\right) \\
\vdots \\
\widehat{\Pi_{1 ; n}}\left(u_{1}, \ldots, u_{k}, v\right)
\end{array}\right)=\left(\begin{array}{c}
\sum_{t=k}^{n} A_{0}(t) \mathbb{1}_{u v} \\
\vdots \\
\sum_{t=k}^{n} A_{d}(t) \mathbb{1}_{u v}
\end{array}\right) .
$$


Consequently, from Equation (4), for any $u_{1}, \ldots, u_{k}, v \in E$ and $t=1, \ldots, n$, the estimator of $\Pi_{\frac{t}{n}}\left(u 1, \ldots, u_{k}, v\right)$ is given by

$$
\widehat{\Pi}_{\frac{t}{n}}\left(u_{1}, \ldots, u_{k}, v\right)=\sum_{i=0}^{d} A_{i}(t) \widehat{\Pi}_{\frac{i}{d} ; n}\left(u_{1}, \ldots, u_{k}, v\right), u_{1}, \ldots, u_{k}, v \in E
$$

with $\widehat{\Pi}_{\frac{i}{d} ; n}(u, v)$ given by solving the system $(23)$.

Second, as in the framework of Proposition 9, let us now consider $H$ i.i.d. sample paths of a polynomial drifting Markov chain of order $k$ and degree $d,\left(X_{k}\right)_{0 \leq k \leq n}$. That is, we assume that the sample paths have the same length $n$ and that the polynomial drift within the $H$ sample paths takes place between the same $d$ Markov transition matrices (of order k) $\Pi_{\frac{i}{d}}$. The proof is similar to proof of Proposition 9.

Proposition 11 Let us note $\mathbb{1}_{u}^{h}=\mathbb{1}_{\left\{X_{t-k}^{h} \ldots X_{t-1}^{h}=u_{1} \ldots u_{k}\right\}}$ and $\mathbb{1}_{u v}^{h}=\mathbb{1}_{\left\{X_{t-k}^{h} \ldots X_{t-1}^{h}=u_{1} \ldots u_{k}, X_{t}^{h}=v\right\}}$. Under this setting, starting with $H$ sample paths of a polynomial drifting Markov chain of order $k$ and degree $d$, for any states $u_{1}, \ldots, u_{k}, v \in E$, the estimators of $\Pi_{\frac{i}{d}}\left(u_{1}, \ldots, u_{k}, v\right)$ are obtained by solving the linear system

$$
\left(\begin{array}{c}
\sum_{t=k}^{n} \sum_{h=1}^{H} \mathbb{1}_{u}^{h} A_{0}(t) A_{0}(t) \cdots \sum_{t=k}^{n} \sum_{h=1}^{H} \mathbb{1}_{u}^{h} A_{0}(t) A_{d}(t) \\
\vdots \\
\sum_{t=k}^{n} \sum_{h=1}^{H} \mathbb{1}_{u}^{h} A_{d}(t) A_{0}(t) \cdots \sum_{t=k}^{n} \sum_{h=1}^{H} \mathbb{1}_{u}^{h} A_{d}(t) A_{d}(t)
\end{array}\right)\left(\begin{array}{c}
\widehat{\Pi_{0 ;(n, H)}}\left(u_{1}, \ldots, u_{k}, v\right) \\
\vdots \\
\widehat{\Pi_{1 ;(n, H)}}\left(u_{1}, \ldots, u_{k}, v\right)
\end{array}\right)=\left(\begin{array}{c}
\sum_{t=k}^{n} A_{0}(t) \sum_{h=1}^{H} \mathbb{1}_{u v}^{h} \\
\vdots \\
\sum_{t=k}^{n} A_{d}(t) \sum_{h=1}^{H} \mathbb{1}_{u v}^{h}
\end{array}\right) .
$$

Proposition 12 For a polynomial drift Markov chain of order $k$, degree $d$ and length $n$ within the $H$ sample paths, the estimators $\widehat{\Pi}_{\frac{i}{d} ;(n, H)}(u, v)$ verify $\sum_{v \in E} \widehat{\Pi}_{\frac{i}{d} ;(n, H)}(u, v)=1$.

\section{Proof.}

From the system of Proposition 11, we provide the following system by summing over $v$ :

$$
\left(\begin{array}{c}
\sum_{t=k}^{n} \sum_{h=1}^{H} \mathbb{1}_{u}^{h} A_{0}(t) A_{0}(t) \cdots \sum_{t=k}^{n} \sum_{h=1}^{H} \mathbb{1}_{u}^{h} A_{0}(t) A_{d}(t) \\
\vdots \\
\sum_{t=k}^{n} \sum_{h=1}^{H} \mathbb{1}_{u}^{h} A_{d}(t) A_{0}(t) \cdots \sum_{t=k}^{n} \sum_{h=1}^{H} \mathbb{1}_{u}^{h} A_{d}(t) A_{d}(t)
\end{array}\right)\left(\begin{array}{c}
\sum_{v \in \mathcal{A}} \widehat{\Pi_{0 ;(n, H)}}(u, v) \\
\vdots \\
\sum_{v \in \mathcal{A}} \widehat{\Pi_{1 ;(n, H)}}(u, v)
\end{array}\right)=\left(\begin{array}{c}
\sum_{t=k}^{n} \sum_{h=1}^{H} A_{0}(t) \mathbb{1}_{u}^{h} \\
\vdots \\
\sum_{t=k}^{n} \sum_{h=1}^{H} A_{d}(t) \mathbb{1}_{u}^{h}
\end{array}\right) .
$$

The solution of this system is $(1, \ldots, 1)^{\top}$.

\subsection{Estimation for other types of data}

As we said at the beginning of this section, different types of data can be considered, that we have denoted by A1, A2, B1, B2 and B3. Note that the estimators of $\boldsymbol{\Pi}_{0}$ and $\boldsymbol{\Pi}_{1}$ in the framework A1 were given in Proposition 8, while in the framework B1 were given in Proposition 9 and their convergence was the object of Theorem 1. In the sequel we will provide the estimators of $\boldsymbol{\Pi}_{0}$ and $\boldsymbol{\Pi}_{1}$ in the other frameworks, namely A2, B2, B3 and B4. The proofs of these results are similar to those of Propositions 8 and 9. For the sake of simplicity, we confine here to a linear drifting Markov model of order 1 . The polynomial and $k$ order Markov case can be considered in a similar way. 
Proposition 13 Under the statistical framework A2, for $\left(X_{0}, X_{1}, \ldots, X_{m}\right), m \leq n$, an incomplete sample path of a linear drifting Markov chain (of order 1 ) of length $n$ between $\boldsymbol{\Pi}_{0}$ and $\boldsymbol{\Pi}_{1}$, for any states $u, v \in E$, the estimators of $\Pi_{0}(u, v)$ and $\Pi_{1}(u, v)$ are given by:

$$
\begin{aligned}
\widehat{\Pi}_{0 ; n}(u, v) & =\frac{P_{1}(1, m, n) P_{2}(1, m, n)-P_{3}(1, m, n) P_{4}(1, m, n)}{P_{5}(1, m, n) P_{1}(1, m, n)-P_{3}(1, m, n)^{2}}, \\
\widehat{\Pi}_{1 ; n}(u, v) & =\frac{P_{5}(1, m, n) P_{4}(1, m, n)-P_{3}(1, m, n) P_{2}(1, m, n)}{P_{5}(1, m, n) P_{1}(1, m, n)-P_{3}(1, m, n)^{2}},
\end{aligned}
$$

with $m \leq n$.

Proposition 14 Under the statistical framework B2, for $\left(X_{0}^{h}, X_{1}^{h}, \ldots, X_{m_{h}}^{h}\right), m_{h} \leq n, h=1, \ldots, H, H$ i.i.d. incomplete sample paths of a linear drifting Markov chain (of order 1) of length $n$ between $\boldsymbol{\Pi}_{0}$ and $\boldsymbol{\Pi}_{1}$, for any states $u, v \in E$, the estimators of $\Pi_{0}(u, v)$ and $\Pi_{1}(u, v)$ are given by:

$$
\begin{aligned}
& \widehat{\Pi}_{0 ;(n, H)}(u, v)=\frac{P_{1}(H, \boldsymbol{m}, \boldsymbol{n}) P_{2}(H, \boldsymbol{m}, \boldsymbol{n})-P_{3}(H, \boldsymbol{m}, \boldsymbol{n}) P_{4}(H, \boldsymbol{m}, \boldsymbol{n})}{P_{5}(H, \boldsymbol{m}, \boldsymbol{n}) P_{1}(H, \boldsymbol{m}, \boldsymbol{n})-P_{3}(H, \boldsymbol{m}, \boldsymbol{n})^{2}}, \\
& \widehat{\Pi}_{1 ;(n, H)}(u, v)=\frac{P_{5}(H, \boldsymbol{m}, \boldsymbol{n}) P_{4}(H, \boldsymbol{m}, \boldsymbol{n})-P_{3}(H, \boldsymbol{m}, \boldsymbol{n}) P_{2}(H, \boldsymbol{m}, \boldsymbol{n})}{P_{5}(H, \boldsymbol{m}, \boldsymbol{n}) P_{1}(H, \boldsymbol{m}, \boldsymbol{n})-P_{3}(H, \boldsymbol{m}, \boldsymbol{n})^{2}},
\end{aligned}
$$

with $\boldsymbol{n}=\left(n_{1}, \ldots, n_{H}\right)=(n, \ldots, n)$ and $\boldsymbol{m}=\left(m_{1}, \ldots, m_{H}\right)$ where $m_{h} \leq n$ for $h=1, \ldots, H$.

Proposition 15 Under the statistical framework B3, for $\left(X_{0}^{h}, X_{1}^{h}, \ldots, X_{n_{h}}^{h}\right), h=1, \ldots, H, H$ i.i.d. complete sample paths of $H$ linear drifting Markov chains (of order 1 ), each of them of length $n_{i}, i=1, \ldots, H$, between the same $\boldsymbol{\Pi}_{0}$ and $\boldsymbol{\Pi}_{1}$, for any states $u, v \in E$, the estimators of $\Pi_{0}(u, v)$ and $\Pi_{1}(u, v)$ are given by:

$$
\begin{aligned}
& \widehat{\Pi}_{0 ;(\boldsymbol{n}, H)}(u, v)=\frac{P_{1}(H, \boldsymbol{n}, \boldsymbol{n}) P_{2}(H, \boldsymbol{n}, \boldsymbol{n})-P_{3}(H, \boldsymbol{n}, \boldsymbol{n}) P_{4}(H, \boldsymbol{n}, \boldsymbol{n})}{P_{5}(H, \boldsymbol{n}, \boldsymbol{n}) P_{1}(H, \boldsymbol{n}, \boldsymbol{n})-P_{3}(H, \boldsymbol{n}, \boldsymbol{n})^{2}}, \\
& \widehat{\Pi}_{1 ;(\boldsymbol{n}, H)}(u, v)=\frac{P_{5}(H, \boldsymbol{n}, \boldsymbol{n}) P_{4}(H, \boldsymbol{n}, \boldsymbol{n})-P_{3}(H, \boldsymbol{n}, \boldsymbol{n}) P_{2}(H, \boldsymbol{n}, \boldsymbol{n})}{P_{5}(H, \boldsymbol{n}, \boldsymbol{n}) P_{1}(H, \boldsymbol{n}, \boldsymbol{n})-P_{3}(H, \boldsymbol{n}, \boldsymbol{n})^{2}},
\end{aligned}
$$

with $\boldsymbol{n}=\left(n_{1}, \ldots, n_{H}\right)$.

Proposition 16 Under the statistical framework $\mathbf{B} 4$, for $\left(X_{0}^{h}, X_{1}^{h}, \ldots, X_{m_{h}}^{h}\right), m_{h} \leq n_{h}, h=1, \ldots, H, H$ i.i.d. incomplete sample paths of $H$ linear drifting Markov chains (of order 1 ), each of them of length $n_{i}, i=1, \ldots, H$, between the same $\boldsymbol{\Pi}_{0}$ and $\Pi_{1}$, for any states $u, v \in E$, the estimators of $\Pi_{0}(u, v)$ and $\Pi_{1}(u, v)$ are given by:

$$
\begin{aligned}
& \widehat{\Pi}_{0 ;(\boldsymbol{n}, H)}(u, v)=\frac{P_{1}(H, \boldsymbol{m}, \boldsymbol{n}) P_{2}(H, \boldsymbol{m}, \boldsymbol{n})-P_{3}(H, \boldsymbol{m}, \boldsymbol{n}) P_{4}(H, \boldsymbol{m}, \boldsymbol{n})}{P_{5}(H, \boldsymbol{m}, \boldsymbol{n}) P_{1}(H, \boldsymbol{m}, \boldsymbol{n})-P_{3}(H, \boldsymbol{m}, \boldsymbol{n})^{2}}, \\
& \widehat{\Pi}_{1 ;(\boldsymbol{n}, H)}(u, v)=\frac{P_{5}(H, \boldsymbol{m}, \boldsymbol{n}) P_{4}(H, \boldsymbol{m}, \boldsymbol{n})-P_{3}(H, \boldsymbol{m}, \boldsymbol{n}) P_{2}(H, \boldsymbol{m}, \boldsymbol{n})}{P_{5}(H, \boldsymbol{m}, \boldsymbol{n}) P_{1}(H, \boldsymbol{m}, \boldsymbol{n})-P_{3}(H, \boldsymbol{m}, \boldsymbol{n})^{2}},
\end{aligned}
$$

with $\boldsymbol{m}=\left(m_{1}, \ldots, m_{H}\right)$ and $\boldsymbol{n}=\left(n_{1}, \ldots, n_{H}\right)$.

As we did before in Equations (17) and (20) for the frameworks A1 and B1, it is clear that in any of these new statistical frameworks, from Equation (2), for any $u, v \in E$ one can immediately obtain an estimator of $\Pi_{\frac{t}{n}}(u, v), t=1, \ldots, n$, for the frameworks $\mathbf{A} 2$ and $\mathbf{B 2}$, respectively of $\Pi_{\frac{t}{n_{h}}}(u, v), t=1, \ldots, n_{h}, h=1, \ldots H$, for the frameworks $\mathbf{B 3}$ and $\mathbf{B} 4$.

\subsection{Estimation of reliability and survival measures}

Using the expression of the reliability indicators of a drifting Markov chain obtained in Subsection 2.2 and the estimators obtained in Subsection 3.2 for the characteristics of a drifting Markov chain, one immediately obtain the associated plug-in estimators. We will write in the sequel these estimators for a linear drifting Markov chain of order 1 , under the statistical framework B1. We will also give some comments in Remark 6 about the associated estimation under the other frameworks. 
Proposition 17 Under the statistical framework B1, for $\left(X_{0}^{h}, X_{1}^{h}, \ldots, X_{n}^{h}\right), h=1, \ldots, H, H$ i.i.d. complete sample paths of a linear drifting Markov chain (of order 1) of length $n$ between $\boldsymbol{\Pi}_{0}$ and $\boldsymbol{\Pi}_{1}$, the estimators of the reliability, availability, maintainability, BMP-failure rate, $R G$-failure rate at time $l, l \in \mathbb{N}$, are given by:

$$
\begin{aligned}
& \widehat{R}_{(n, H)}(l)=\widehat{\boldsymbol{\alpha}}_{H}^{U} \prod_{t=1}^{l}\left(\left(1-\frac{t}{n}\right) \widehat{\boldsymbol{\Pi}}_{0 ;(n, H)}^{U U}+\frac{t}{n} \widehat{\boldsymbol{\Pi}}_{1 ;(n, H)}^{U U}\right) \mathbb{1}^{U}, \\
& \widehat{A}_{(n, H)}(l)=\widehat{\boldsymbol{\alpha}}_{H} \prod_{t=1}^{l}\left(\left(1-\frac{t}{n}\right) \widehat{\boldsymbol{\Pi}}_{0 ;(n, H)}+\frac{t}{n} \widehat{\boldsymbol{\Pi}}_{1 ;(n, H)}\right) \mathbb{1}^{E, U}, \\
& \widehat{M}_{(n, H)}(l)=1-\widehat{\boldsymbol{\alpha}}_{H}^{D} \prod_{t=1}^{l}\left(\left(1-\frac{t}{n}\right) \widehat{\boldsymbol{\Pi}}_{0 ;(n, H)}^{D D}+\frac{t}{n} \widehat{\boldsymbol{\Pi}}_{1 ;(n, H)}^{D D}\right) \mathbb{1}^{D}, \\
& \widehat{\lambda}_{(n, H)}(l)= \begin{cases}1-\frac{\widehat{R}_{(n, H)}(l)}{\widehat{R}_{(n, H)}(l-1)}, & \text { if } \widehat{R}_{(n, H)}(l-1) \neq 0, \\
0, & \text { otherwise, }\end{cases} \\
& \widehat{r}_{(n, H)}(l)= \begin{cases}-\ln \frac{\widehat{R}_{(n, H)}(l)}{\widehat{R}_{(n, H)}(l-1)}, \text { if } l \geq 1, \\
-\ln \widehat{R}_{(n, H)}(0), & \text { if } l=0,\end{cases}
\end{aligned}
$$

for $\widehat{R}_{(n, H)}(l) \neq 0$ (if $\widehat{R}_{(n, H)}(l)=0$, we set $\left.\widehat{r}_{(n, H)}(l):=0\right)$, where, for any states $u, v \in E$, the estimators $\widehat{\alpha}_{H}(u), \widehat{\Pi}_{0 ;(n, H)}(u, v)$, $\widehat{\Pi}_{1 ;(n, H)}(u, v)$ were obtained in Equations (22), (18) and (19), $\mathbb{1}^{U}=(\underbrace{1, \cdots, 1}_{s_{1}})^{\top}, \mathbb{1}^{E, U}=(\underbrace{1, \cdots, 1}_{s_{1}}, \underbrace{0, \cdots, 0}_{s-s_{1}})^{\top}, \mathbb{1}^{D}=$ $(\underbrace{1, \cdots, 1}_{s-s_{1}})^{\top}$

Using the strong consistency of the estimators $\widehat{\Pi}_{0 ;(n, H)}(u, v)$ and $\widehat{\Pi}_{1 ;(n, H)}(u, v)$ for any states $u, v \in E$, as $H$ goes to infinity (cf. Theorem 2), and the continuous mapping theorem, we immediately obtain the strong consistency of the estimators of reliability indicators given in Proposition 17. These facts are stated in the following result.

Theorem 2 Under the statistical framework $\mathbf{B 1}$, for $\left(X_{0}^{h}, X_{1}^{h}, \ldots, X_{n}^{h}\right), h=1, \ldots, H, H$ i.i.d. complete sample paths of a linear drifting Markov chain (of order 1) of length n between $\boldsymbol{\Pi}_{0}$ and $\boldsymbol{\Pi}_{1}$, the estimators of the reliability, availability, maintainability, BMP-failure rate, $R G$-failure rate at time $l, l \in \mathbb{N}$, given in Proposition 17 are strongly consistent, i.e.,

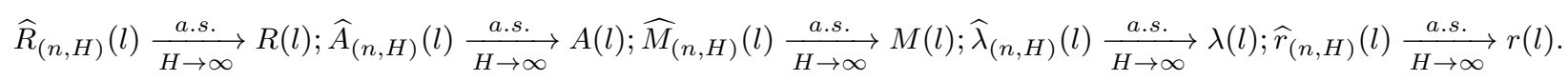

Remark 6 In Proposition 17 we considered the estimators of the reliability indicators under the statistical framework B1, that is starting with several complete sample paths of a linear drifting Markov chain (of order 1) of the same length between $\boldsymbol{\Pi}_{0}$ and $\boldsymbol{\Pi}_{1}$. We would like to give some comments about the other statistical frameworks.

1. Note that the initial distribution $\boldsymbol{\alpha}$ can be estimated only if we have at our disposal several sample paths of the drifting Markov chain (the statistical frameworks B1-B4). In the other cases, one can consider that the initial distribution is known (given), as it is the case when we know that the system starts in a perfect working state. Another possibility is to consider that the initial distribution is the stationary distribution of the Markov transition matrix $\boldsymbol{\Pi}_{0}$.

2. On the one hand, if we are under the statistical frameworks A1, A2 or B2, we can obtain the estimators of the reliability indicators as we did in Proposition 17 for the statistical framework B1, by considering the estimators of $\boldsymbol{\Pi}_{0}$ and $\boldsymbol{\Pi}_{1}$ given in Propositions 8, 13 and 14, respectively.

3. On the other hand, if we are under the statistical frameworks B3 and B4, obviously that we can estimate the transition matrices $\boldsymbol{\Pi}_{0}$ and $\boldsymbol{\Pi}_{1}$ using Propositions 15 and 16, respectively. Note nonetheless that these two frameworks assume that we start from data issued from different linear drifting Markov chains (consequently, of different theoretical lengths) between the same $\boldsymbol{\Pi}_{0}$ and $\boldsymbol{\Pi}_{1}$. Thus, in order to obtain the corresponding plug-in estimators of the reliability 
indicators, one has to consider (fix) a certain value of the length $n$ of the drifting model of interest (the one that appears in formulas like Formula (5) for the reliability).

\section{Numerical example}

In this section we carry out numerical experiments in order to illustrate the quality of the proposed estimators and to numerically validate our procedures. Thus, in Section 4.1 we investigate the quality of the estimators of $\Pi_{0}$ and $\Pi_{1}$. Then we present the estimators of reliability indicators in Section 4.2. We conclude by retrieving results on reliability of discrete-time Markov systems obtained in [32] as a particular case of our model.

Let us consider a system with state space $E=\{1,2,3,4\}$, partitioned into the up-states $U=\{1,2,3\}$ and down-states $D=\{4\}$. In order to undertake numerical illustration of the results presented in this paper, we consider four different linear drifting models, $M^{(1)}, M^{(2)}, M^{(3)}$ and $M^{(4)}$, on the state space $E$, each of them varying between $\Pi_{0}^{(k)}$ and $\Pi_{1}^{(k)}$, $k=1,2,3,4$. We consider the following numerical examples for the couples of Markov transition matrices:

$$
\begin{aligned}
& \Pi_{0}^{(1)}=\left(\begin{array}{cccc}
0.1 & 0.2 & 0.6 & 0.1 \\
0.5 & 0.1 & 0.2 & 0.2 \\
0.4 & 0.2 & 0.4 & 0 \\
0.05 & 0.05 & 0 & 0.9
\end{array}\right) \text { and } \Pi_{1}^{(1)}=\left(\begin{array}{cccc}
0.2 & 0.1 & 0.7 & 0 \\
0.8 & 0.1 & 0 & 0.1 \\
0.35 & 0.4 & 0.2 & 0.05 \\
0.1 & 0.1 & 0.1 & 0.7
\end{array}\right) \\
& \Pi_{0}^{(2)}=\left(\begin{array}{llll}
0.2 & 0.2 & 0.3 & 0.3 \\
0.1 & 0.4 & 0.4 & 0.1 \\
0.4 & 0.1 & 0.4 & 0.1 \\
0.3 & 0.3 & 0.2 & 0.2
\end{array}\right) \text { and } \Pi_{1}^{(2)}=\left(\begin{array}{llll}
0.4 & 0.1 & 0.4 & 0.1 \\
0.3 & 0.3 & 0.2 & 0.2 \\
0.2 & 0.2 & 0.3 & 0.3 \\
0.1 & 0.4 & 0.4 & 0.1
\end{array}\right) ;
\end{aligned}
$$

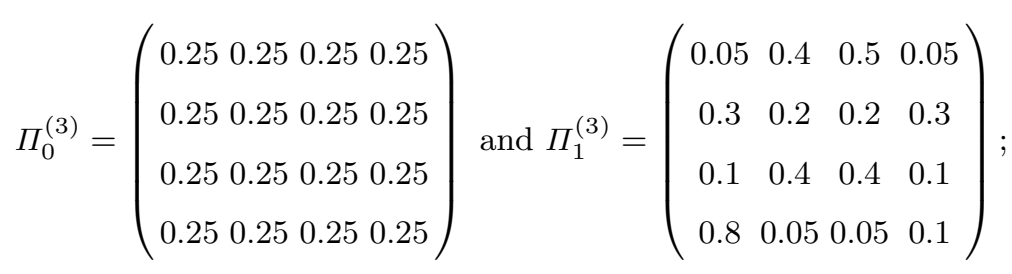

$$
\begin{aligned}
& \Pi_{0}^{(4)}=\left(\begin{array}{cccc}
0.01 & 0.1 & 0.09 & 0.8 \\
0.8 & 0.09 & 0.01 & 0.1 \\
0.7 & 0.1 & 0.1 & 0.1 \\
0.1 & 0.1 & 0.7 & 0.1
\end{array}\right) \text { and } \Pi_{1}^{(4)}=\left(\begin{array}{cccc}
0.8 & 0.09 & 0.01 & 0.1 \\
0.1 & 0.09 & 0.01 & 0.8 \\
0.1 & 0.1 & 0.1 & 0.7 \\
0.1 & 0.7 & 0.1 & 0.1
\end{array}\right) .
\end{aligned}
$$

\subsection{Convergence of the estimators}

In order to illustrate the convergence of the estimators of $\Pi_{0}$ and $\Pi_{1}$ as the number of sequences increases, in according to the results obtained in Theorem 1 , we simulate $H$ sequences $(H=5,10$ and 100) of length $n=100$ for each of the four different models considered, $M^{(i)}, i=1, \ldots, 4$. We compute the following distance $d$ between each matrix of parameters $\Pi_{i}^{(k)}$ and their estimators $\widehat{\Pi}_{i}^{(k)}, i=0,1, k=1, \ldots, 4$ :

$$
d\left(\Pi_{i}^{(k)}, \widehat{\Pi}_{i ;(n, H)}^{(k)}\right)=\sum_{u, v \in E}\left(\Pi_{i}^{(k)}(u, v)-\widehat{\Pi}_{i ;(n, H)}^{(k)}(u, v)\right)^{2}
$$


The obtained distances are given in Table 1 . We observe a convergence with the number of sequences $H$ for all the models, fact that is in accordance with Theorem 1. Note that even for small values of $H$ we have excellent results for the convergence.

\begin{tabular}{|c|c|c|c|}
\hline Model & $H$ & $\Pi_{0}$ & $\Pi_{1}$ \\
\hline \multirow{3}{*}{$M^{(1)}$} & 5 & 0.0001249 & 0.0000496 \\
& 10 & 0.0001058 & 0.00003858 \\
& 100 & 0.000004377 & 0.000002932 \\
\hline \multirow{3}{*}{$M^{(2)}$} & 5 & 0.009501 & 0.003593 \\
& 10 & 0.00304 & 0.002224 \\
& 100 & 0.0001349 & 0.0002502 \\
\hline \multirow{3}{*}{$M^{(3)}$} & 5 & 0.005092 & 0.002636 \\
& 10 & 0.001491 & 0.0008556 \\
& 100 & 0.0000884 & 0.0001761 \\
\hline \multirow{3}{*}{$M^{(4)}$} & 5 & 0.001448 & 0.000993 \\
& 10 & 0.0006423 & 0.0004372 \\
& 100 & 0.00006185 & 0.00009181 \\
\hline
\end{tabular}

Table 1 Convergence of the estimators: different values of the distance (39)

\subsection{Reliability measures}

In order to observe the convergence of the estimators of reliability measures as the number of sequences $H$ increases (Theorem 2), we simulate $H$ sequences $\left(H=5,10\right.$ and 100) of length $n=1000$, according to the model $M^{(1)}$.

In Figure 1 we have represented the reliability function as well as the corresponding estimators obtained for these values of $H$. Notice that the estimator approaches the true value as the number of sequences increases, which is in accordance with Theorem 2.

Similarly, in Figure 2 we have represented the availability function as well as the corresponding estimators, while in Figure 3 we have represented the classical failure rate function and associated estimators. Notice that we represented the entire length of the trajectory and also a zoom up to time 30 .

We can notice that the estimator approaches the true value as the number of sequences increases, in accordance with Theorem 2. Note also that in both entire figures we observe the drift between the two Markov models.

\subsection{Specification to Markov case}

We would like also to considered the particular case where $\Pi_{0}$ and $\Pi_{1}$ are the same, equal to $\Pi_{1}^{(1)}$ given before. In other words, this is the classical discrete time Markov system for which reliability measures and associated estimation have been investigated in [32].

In Figure 4 we have represented Reliability, Availability and Failure Rate together with the corresponding estimators obtained for a number of trajectories $H=5,10$ and 100. Here, we represented only up to time 50 .

We can notice that the obtain results are in agreement with those presented in [32]. 


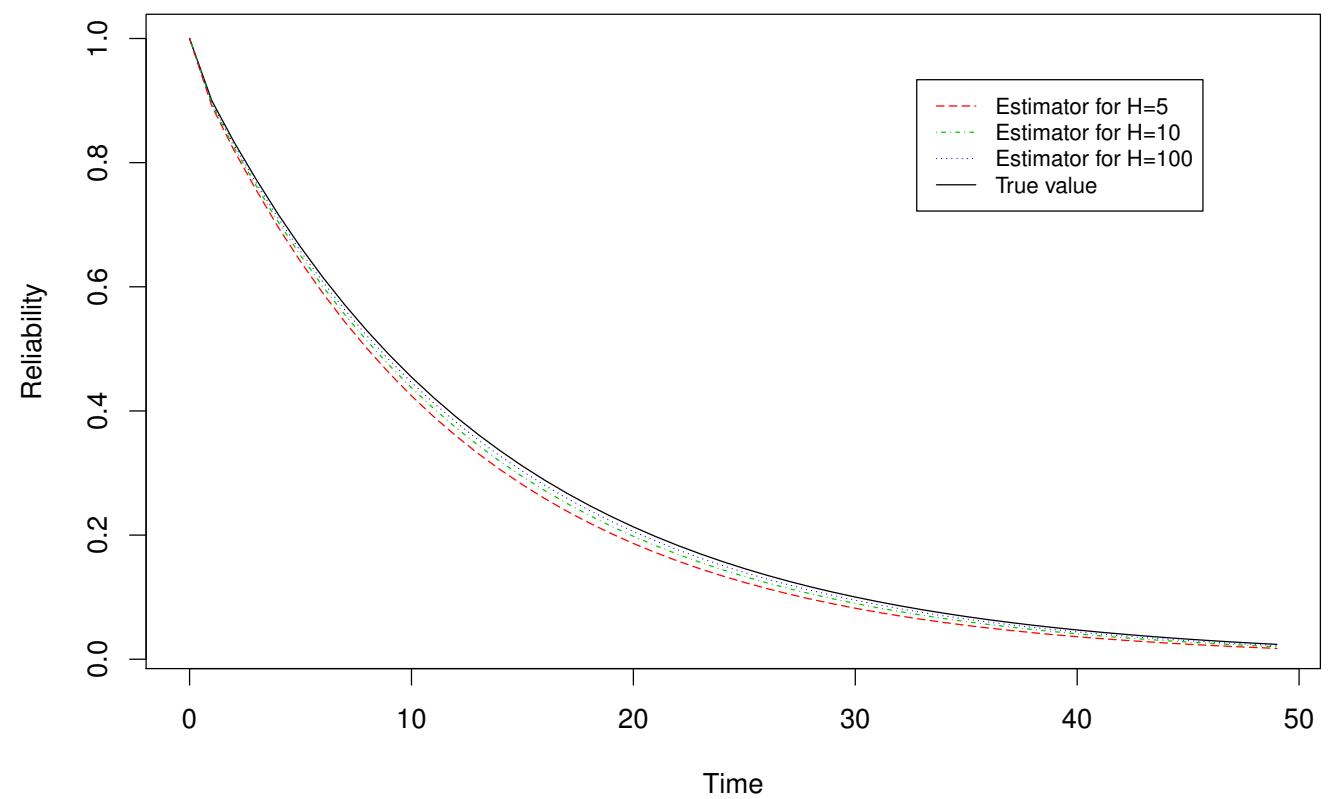

Fig. 1 Reliability estimation
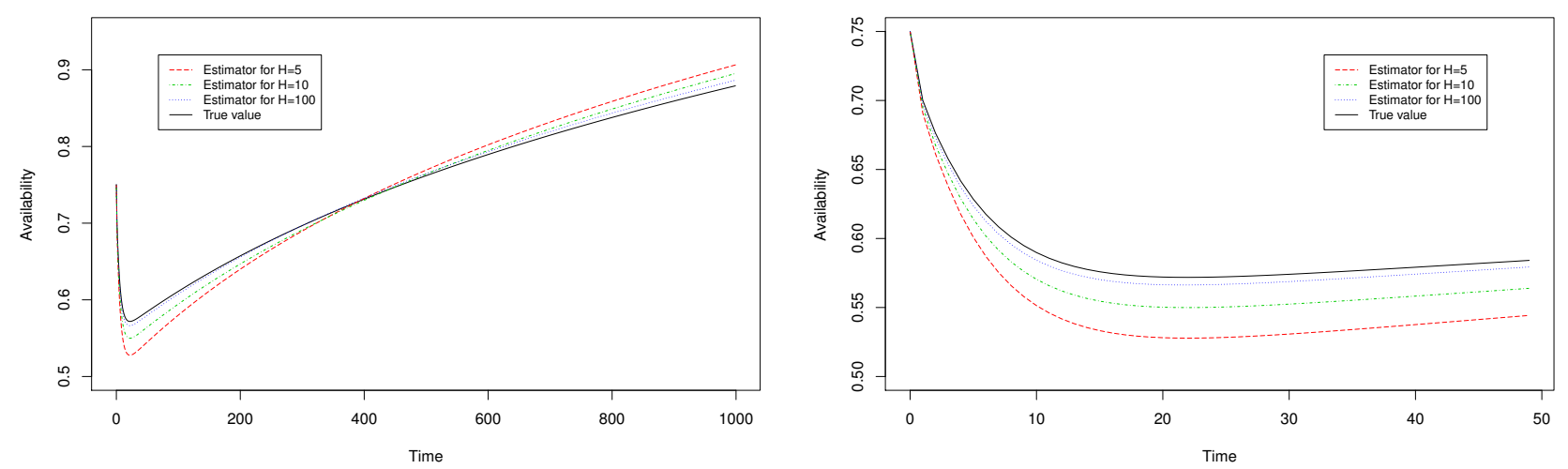

Fig. 2 Availability estimation: all the trajectory (left) and a zoom (right)
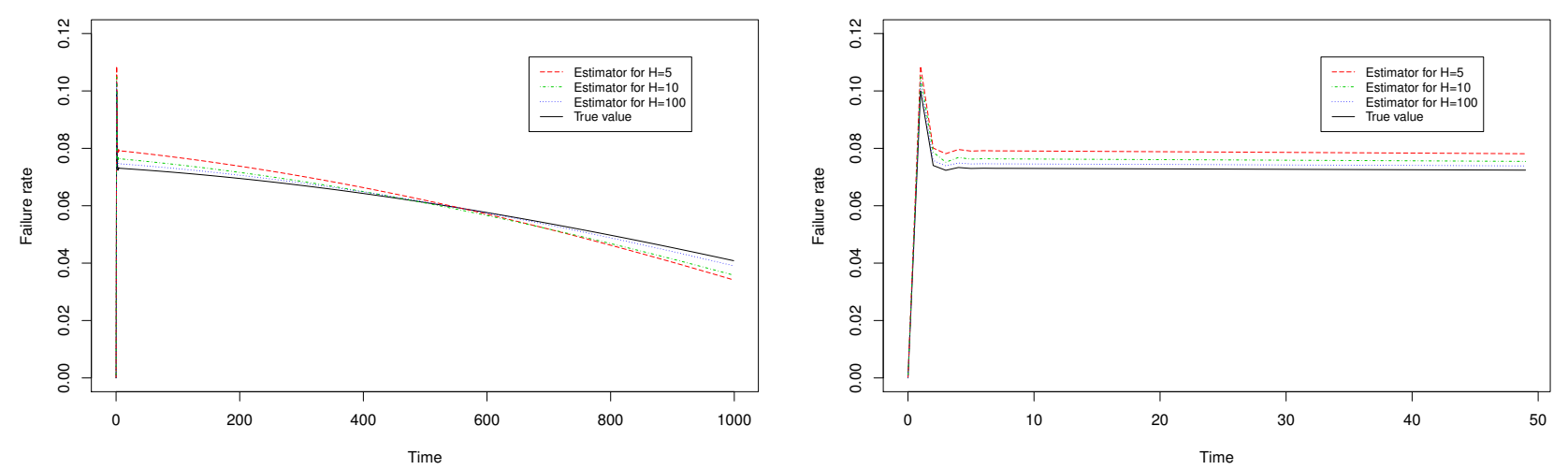

Fig. 3 Failure rate estimation: all the trajectory (up) and a zoom (down) 

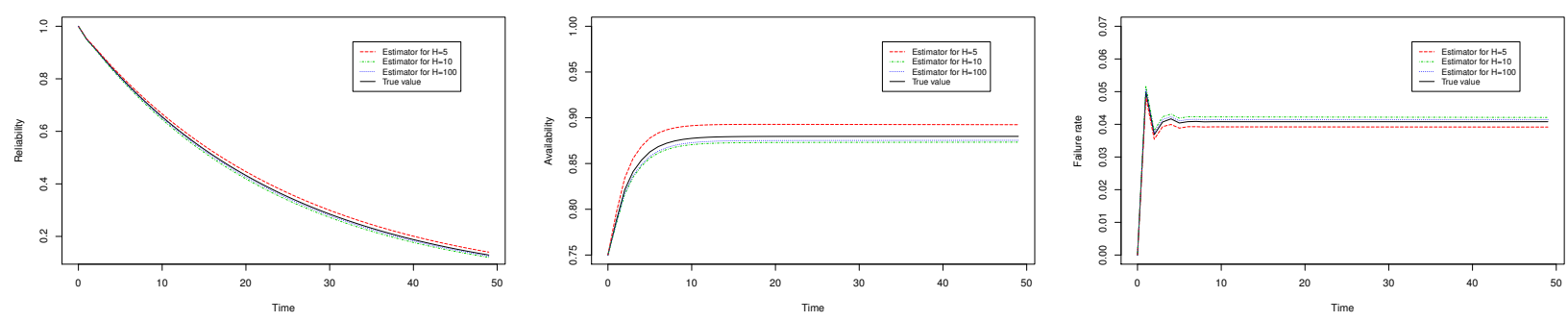

Fig. 4 Reliability, Availability and Failure Rate 


\section{Concluding Remarks}

In this article we have used for the first time in reliability theory the particular non-homogeneous Markov model called drifting Markov model. We took into account different types of drifting Markov chains, allowing for general order of the Markov chains and also for polynomial drifting. Explicit expressions for reliability measures were obtained and estimators of the basic quantities and of the reliability indicators are derived. Note that we considered various statistical frameworks that could be important in different practical situations.

The main novelty of the present article comes from proposing in reliability theory a particular case of non-homogeneous Markov model that is obtain through a gradual variation between two or more Markov models. The type of variation we considered ranges from the simplest linear one to a general polynomial.

Several directions for further research can be investigated.

A natural ongoing research direction is developing analogous notions of semi-Markov drifting models and carrying out applications of this kind of models, for instance in reliability theory.

Also another natural research direction is to consider continuous-time Markov drifting models. It is worth mentioning here the importance of having both developments of discrete-time and continuous-time drifting Markov models. On the one hand, note that continuous-time models are those that are used in most of the applications. On the other hand, note that there is also an intrinsic interest in considering discrete-time models (along with continuous-time versions of these models). Indeed, there are applications (in reliability and survival among others) where the time is discrete by nature; we think about the cycles of an industrial application, the various applications with counting processes, the DNA applications, etc. As we have different theories of discrete-time and continuous-time Markov processes, in our opinion it is of interest to have different developments of discrete-time and continuous-time drifting Markov models. Moreover, the techniques used in discrete and continuous time are different.

Another direction is considering an infinite countable state space for the drifting Markov chains ; generally, in reliability/survival analysis studies (in MSS studies in general) the state space is assumed to be finite. Nonetheless, there could be situations where one could be interested in considering systems with infinite number of states. Obviously, although in practice we always deal with finite quantities (states, number of performance rates, etc.), it could happen that this number of states is not necessarily known in advance. Thus a general and unifying framework could be to consider an infinite countable state space for drifting Markov chains and to develop associated probabilistic/statistical theory.

Nonetheless, several problems may arise when working with infinite matrices: the matrix multiplication is not always defined (for obvious reasons of series convergence), it is not always associative, and the existence of left and right inverse is not an obvious matter in the general case. The reader interested in infinite matrices can see the references and discussions in [7], that deals with countable state space semi-Markov chains.

Finally, a more advanced research direction could be to consider that the drifting system has continuous-state space. For continuous-state systems, one can refer to [22] and the references within. This case could be important for applications where the scale of the measures is continuous. As in practice continuous-state systems are tractable with difficulty, people generally do discretizations, by considering associated discrete-state systems instead of continuous-state systems. One can see [24] for such an application for the reliability of a coal power generating unit. 


\section{Acknowledgments}

This research work was partially supported by the projects MMATFAS - Mathematical Methods for System Reliability and Survival Analysis (2014-2015) and MOUSTIC-Random Models and Statistical, Informatics and Combinatorics Tools (20162019), within the Large Scale Research Networks from the Region of Normandy, France.

The authors would like to thank their colleagues Dr. Anatoly Lisnianski and Dr. Gregory Levitin from the Israel Electric Corporation Ltd. for pointing out important aspects and references on multi-state systems and also to Prof. Ilia Frenkel and his colleagues from Sami Shamoon College of Engineering, Israel, for organizing the series of conferences International Symposium on Stochastic Models in Reliability Engineering, Life Science and Operations Management, to which, indirectly, the research developed in this article owes much.

\section{References}

1. Alvarez EE (2005) Estimation in stationary Markov renewal processes, with application to earthquake forecasting in Turkey. Methodology and Computing in Applied Probability 7(1): 119-130. doi: 10.1007/s11009-005-6658-2

2. Barbu VS, Karagrigoriou A, Makrides A (2016) Semi-Markov modelling for multi-state systems. Methodology and Computing in Applied Probability 19: 1011-1028. doi: 10.1007/s11009-016-9510-y

3. Barbu VS, Limnios N (2004) Discrete time semi-Markov processes for reliability and survival analysis-a nonparametric estimation approach. In Balakrishnan N, Nikulin M, Mesbah M, Limnios N (eds) Parametric and semiparametric models with applications to reliability, survival analysis and quality of life, Collection Statistics for Industry and Technology. Birkhäuser, Boston, pp 487-502

4. Barbu VS, Limnios N (2006) Nonparametric estimation for failure rate functions of discrete time semi-Markov processes. In Nikulin M, Commenges D, Huber-Carol C (eds) Probability, statistics and modelling in public health. Springer, pp 53-72

5. Barbu VS, Limnios N (2008) Reliability of semi-Markov systems in discrete time: modeling and estimation, In Misra KB (ed) Handbook on performability engineering. Springer, pp 369-380

6. Barbu VS, Limnios N (2008) Semi-Markov chains and hidden semi-Markov models toward applications-their use in reliability and DNA analysis. Lecture Notes in Statistics, vol. 191, Springer, New York

7. Barbu VS, Limnios N (2010) Some algebraic methods in semi-Markov processes. In: Viana MAG, Wynn HP (eds) Algebraic methods in statistics and probability, vol 2, series Contemporary Mathematics edited by AMS. Urbana, pp 19-35

8. Barlow RE, Marshall AW, Prochan F (1963) Properties of probability distributions with monotone hazard rate. Ann Math Statist 34: 375-389

9. Cappé O, Moulines E, Ryden T (2005) Inference in hidden Markov models. Springer, New York

10. Chiquet J, Limnios N (2006) Estimating stochastic dynamical systems driven by a continuous-time jump Markov process. Methodology and Computing in Applied Probability 8(4): 431-447. doi: 10.1007/s11009-006-0423-z

11. Chryssaphinou O, Limnios N, Malefaki S (2011) Multi-state reliability systems under discrete time semi-Markovian hypothesis. IEEE Transactions on Reliability 60(1): 80-87. doi: 10.1109/TR.2010.2104210

12. D'Amico G, Gismondi F, Janssen J, Manca R (2015) Discrete time homogeneous Markov processes for the study of the basic risk processes. Methodology and Computing in Applied Probability 17(4): 983-998. doi: 10.1007/s11009-014-9416-5

13. D'Amico G, Petroni F, Prattico F (2015) Performance analysis of second order semi-Markov chains: an application to wind energy production. Methodology and Computing in Applied Probability 17(3): 781-794. doi: 10.1007/s11009-013-9394-z

14. Hou Y, Limnios N, Schön W (2017) On the existence and uniqueness of solution of MRE and applications. Methodology and Computing in Applied Probability. doi: 10.1007/s11009-017-9570-7

15. Kemeny JG, Snell JL, Knapp AW (1976) Denumerable Markov chains, 2nd edn, series Graduate Texts in Mathematics, vol. 40, Springer, New York

16. Koutras VP, Malefaki S, Platis AN (2017) Optimization of the dependability and performance measures of a generic model for multistate deteriorating systems under maintenance. Reliability Engineering and System Safety 166: 73-86

17. Levitin G (2005) The universal generating function in reliability analysis and optimization. Springer, London

18. Limnios N, Oprişan G (2001) Semi-Markov processes and reliability. Birkhäuser, Boston

19. Limnios N, Ouhbi B (2003) Empirical estimators of reliability and related functions for semi-Markov systems. In: Lindqvist BH, Doksum KA (eds) Mathematical and statistical methods in reliability, 7. World Scientific, Singapore, pp 469-484 
20. Limnios N, Ouhbi B (2006) Nonparametric estimation of some important indicators in reliability for semi-Markov processes. Stat Methodol 3: 341-350

21. Limnios N (2012) Reliability measures of semi-Markov systems with general state space. Methodology and Computing in Applied Probability 14(4): 895-917. doi: 10.1007/s11009-011-9211-5

22. Lisnianski A (2001) Estimation of boundary points for continuum-state system reliability measures. Reliability Engineering and System Safety 74: $81-88$

23. Lisnianski A, Frenkel I, Ding Y (2010) Multi-state system reliability analysis and optimization for engineers and industrial managers. Springer, London

24. Lisnianski A, Elmakias D, Laredo D, Ben Haim H (2012) A multi-state Markov model for a short-term reliability analysis of a power generating unit. Reliability Engineering and System Safety 98(1): 1-6

25. Lisnianski A, Levitin G (2003) Multi-state system reliability: assessment, optimization and applications. World Scientific, Singapore

26. MacDonald IL, Zucchini W (1997) Hidden Markov and other models for discrete-valued time series. Chapman and Hall, London

27. Mathieu E, Foucher Y, Dellamonica P, Daures JP (2007) Parametric and non homogeneous semi-Markov process for HIV control. Methodology and Computing in Applied Probability 9(3): 389-397. doi: 10.1007/s11009-007-9033-7

28. Natvig B (2011) Multistate systems reliability. Theory with applications. Wiley, New York,

29. Ouhbi B, Limnios N (1999) Non-parametric estimation for semi-Markov processes based on its hazard rate functions. Statist Infer Stoch Processes 2(2): 151-173

30. Roy D, Gupta R (1992) Classification of discrete lives. Microelectron Reliabil 32(10): 1459-1473

31. Ruiz-Castro JE (2016) Markov counting and reward processes for analysing the performance of a complex system subject to random inspections. Reliability Engineering and System Safety 145: 155-168

32. Sadek A, Limnios N (2002) Asymptotic properties for maximum likelihood estimators for reliability and failure rates of Markov chains. Comm Statist Theory Methods 31(10): 1837-1861

33. Sadek A, Limnios N (2005) Nonparametric estimation of reliability and survival function for continuous-time finite Markov processes. $\mathrm{J}$ Statistical Planning and Inference 133(1): 1-21

34. Silvestrov D, Manca R (2017) Reward algorithms for semi-Markov processes. Methodology and Computing in Applied Probability. doi: $10.1007 / \mathrm{s} 11009-017-9559-2$

35. Vasiliadis G, Tsaklidis G (2008) On the distributions of the state sizes of closed continuous time homogeneous Markov systems. Methodology and Computing in Applied Probability 11(4): 561-582. doi: 10.1007/s11009-008-9074-6

36. Vassiliou, P-C G, Moysiadis TP (2010) G-inhomogeneous Markov systems of high order. Methodology and Computing in Applied Probability 12(2): 271-292. doi: 10.1007/s11009-009-9143-5

37. Vergne N (2008) Drifting Markov models with polynomial drift and applications to DNA sequences. Statistical Applications in Genetics Molecular Biology $7(1)$

38. Votsi I, Limnios N, Tsaklidis G, Papadimitriou E (2012) Estimation of the expected number of earthquake occurrences based on semi-Markov models. Methodology and Computing in Applied Probability 14 (3): 685-703. doi: 10.1007/s11009-011-9257-4

39. Xie M, Gaudoin O, Bracquemond C (2002) Redefining failure rate function for discrete distributions. Int J Reliabil, Qual, Safety Eng $9(3): 275-285$ 